\title{
Über die sensiblen Wadennerven bei den japanischen Zwillingen.
}

\author{
Von \\ E. Mogi. \\ Aus dem Anatomischen Institut der Keio Universität, Tokyo. \\ (Direktor: Prof. Dr. T. Taniguchi)
}

Mit 45 Textfiguren.

Inhalt.
I. Einleitung . . . . . . . . . . . . . . . . . . 229
II. Naterial und Nethode . . . . . . . . . . . . . . 230
III. Eigene Befunde. . . . . . . . . . . . . . . . . 232

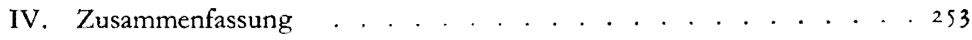
V. Schluss . . . . . . . . . . . . . . 265

\section{Einleitung.}

Morphologische und vergleichend-anatomische L'ntersuchungen über die sensiblen Wadennerven sind seit jeher von vielen Autoren, Thomson (1892), Bardeleben (1906), Gluschkow (I918), Catania (1924), Kosinski (1926), Andreassi (1931), Ssokolow (1933), Ushio (1934), P'an Ming-Tzu (1934) u.a. angestellt worden. Wir finden aber nur wenige Arbeiten bei den Feten, welche die Art und Weise der Nervenverteilung auf dem Wadenteil, d.h. die Verästelungen, Anastomosen oder Dickenverhältnisse der beiden Wadennerven (N. cutaneus surae lateralis und medialis) eingehend studierten. Ferner kennen wir so gut wie keine Befunde in diesem Gebiete bei den Zwillingsfeten, was wahrscheinlich darauf beruht, dass die Ansammlung einer Anzahl Leichen sehr schwerig ist. Um diese Lücke der Untersuchung auszufüllen und eben dadurch zur Kenntnis der Vererbung beizutragen, habe ich die vorliegenden Untersuchungen unternommen. 


\section{Material und Methode.}

Das Untersuchungsmaterial stammte aus 45 paarigen Zwillingsfeten (90 Feten), die im unseren Institute angesammelt worden waren. Sie setzen sich

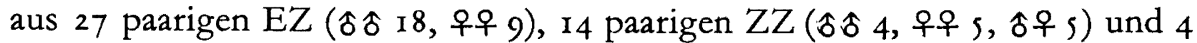
Paaren ( $\hat{o} \hat{o} 4$ ) von unklarer Eiigkeit zusammen, also insgesamt 55 ô und 35 우. Näheres darüber ist aus der Tabelle I ersichtlich.

Die meisten Leichen wurden mit 10\% Formolalkohol im ganzen Körper injiziert und in 10\% Formollösung konserviert.

Ich habe die Untersuchung der Wadennerven mit dem üblichen Präparierungsverfahren vollzogen. Die Befunde wurden alle möglichst genau aufgezeichnet, und sind am Ende dieser Mitteilung zusammengestellt angegeben.

Tabelle I. Untersuchungsmaterial.

\begin{tabular}{|c|c|c|c|c|c|c|}
\hline Fall & Eiigkeit & Leichen-Nr. & Geschlecht & Rumpflänge & $\begin{array}{l}\text { Körper- } \\
\text { gewicht }\end{array}$ & Fetalmonat \\
\hline$x$ & $\mathrm{EZ}$ & $\begin{array}{l}168(\mathrm{I}) \\
\mathrm{I} 68 \text { (II) }\end{array}$ & † & $\begin{array}{l}10.5 \mathrm{~cm} \\
11.0 \\
\end{array}$ & Unklar & 7 \\
\hline 2 & EZ & $\begin{array}{l}228 \text { (I) } \\
228 \text { (II) }\end{array}$ & 字 & $\begin{array}{l}10.5 \\
10.0 \\
\end{array}$ & $\begin{array}{l}700 \mathrm{gr} \\
500\end{array}$ & 7 \\
\hline 3 & $\mathrm{EZ}$ & $\begin{array}{r}322 \\
323\end{array}$ & 우 & $\begin{array}{r}10.3 \\
-9.3\end{array}$ & $\begin{array}{l}570 \\
470 \\
\end{array}$ & 6 \\
\hline 4 & $\mathrm{EZ}$ & $\begin{array}{r}399 \\
400 \\
\end{array}$ & 今 & $\begin{array}{l}\text { II.O } \\
\text { II.S }\end{array}$ & $\begin{array}{r}490 \\
485\end{array}$ & 6 \\
\hline 5 & EZ & $\begin{array}{l}566 \\
567 \\
\end{array}$ & 今 & $\begin{array}{l}13.7 \\
13.5\end{array}$ & $\begin{array}{l}2070 \\
1830\end{array}$ & 9 \\
\hline 6 & $\mathrm{EZ}$ & $\begin{array}{l}734 \\
735\end{array}$ & 우 & $\begin{array}{l}11.2 \\
10.8 \\
\end{array}$ & $\begin{array}{l}550 \\
700\end{array}$ & 7 \\
\hline 7 & $\mathrm{EZ}$ & $\begin{array}{l}768 \\
770 \\
\end{array}$ & 우 & $\begin{array}{r}12.7 \\
13.6\end{array}$ & $\begin{array}{l}1150 \\
1320 \\
\end{array}$ & 8 \\
\hline 8 & $\mathrm{EZ}$ & $\begin{array}{l}825 \\
826 \\
\end{array}$ & 우 & $\begin{array}{l}8.2 \\
6.5 \\
\end{array}$ & $\begin{array}{r}300 \\
\times 70 \\
\end{array}$ & 5 \\
\hline 9 & $\mathrm{EZ}$ & $\begin{array}{l}1012 \\
1013\end{array}$ & 우 & $\begin{array}{l}14.0 \\
14.0 \\
\end{array}$ & $\begin{array}{l}\text { I I } 20 \\
\text { I475 } \\
\end{array}$ & 7 \\
\hline I0 & $\mathrm{EZ}$ & $\begin{array}{l}1063 \\
1064 \\
\end{array}$ & ㅇ & $\begin{array}{l}9.5 \\
6.5\end{array}$ & $\begin{array}{l}670 \\
280\end{array}$ & 6 \\
\hline IX & $\mathrm{EZ}$ & $\begin{array}{l}1107 \\
1108 \\
\end{array}$ & 今 & $\begin{array}{l}15.0 \\
15.0 \\
\end{array}$ & $\begin{array}{l}1875 \\
1650\end{array}$ & 9 \\
\hline 12 & $\mathrm{EZ}$ & $\begin{array}{r}1254 \\
1255 \\
\end{array}$ & $\delta$ & $\begin{array}{r}14.7 \\
15.0 \\
\end{array}$ & $\begin{array}{r}1470 \\
1635\end{array}$ & 8 \\
\hline 13 & $\mathrm{EZ}$ & $\begin{array}{l}1261 \\
1262 \\
\end{array}$ & 우 & $\begin{array}{l}\text { I } 2.2 \\
\text { II.8 }\end{array}$ & $\begin{array}{r}1030 \\
930 \\
\end{array}$ & 7 \\
\hline 14 & $\mathrm{EZ}$ & $\begin{array}{l}1300 \\
1301 \\
\end{array}$ & 今 & $\begin{array}{l}14.0 \\
14.0 \\
\end{array}$ & $\begin{array}{l}1580 \\
1330\end{array}$ & 9 \\
\hline Is & $\mathrm{EZ}$ & $\begin{array}{l}1424 \\
1425 \\
\end{array}$ & $\hat{\delta}$ & $\begin{array}{l}10.5 \\
10.5\end{array}$ & $\begin{array}{l}720 \\
630 \\
\end{array}$ & 7 \\
\hline 16 & $\mathrm{EZ}$ & $\begin{array}{r}1431 \\
1432 \\
\end{array}$ & 우 & $\begin{array}{l}13.8 \\
13.0\end{array}$ & $\begin{array}{r}1250 \\
995 \\
\end{array}$ & 7 \\
\hline 17 & $\mathrm{EZ}$ & $\begin{array}{l}1445 \\
1446 \\
\end{array}$ & $\delta$ & $\begin{array}{l}13.0 \\
13.5 \\
\end{array}$ & $\begin{array}{l}1120 \\
1560 \\
\end{array}$ & 9 \\
\hline I 8 & $\mathrm{EZ}$ & $\begin{array}{l}1453 \\
1454\end{array}$ & $\delta$ & $\begin{array}{r}14.0 \\
12.5 \\
\end{array}$ & $\begin{array}{l}1140 \\
1070\end{array}$ & 8 \\
\hline
\end{tabular}


Tabelle 1.-(Fortsetzung)

\begin{tabular}{|c|c|c|c|c|c|c|}
\hline Fall & Eiigkeit & Leichen-Nr. & Geschlecht & Rumpflänge & $\begin{array}{l}\text { Körper- } \\
\text { gewicht }\end{array}$ & Fetalmonat \\
\hline 19 & $\mathrm{EZ}$ & $\begin{array}{r}1457 \\
1458 \\
\end{array}$ & 우 & $\begin{array}{l}12.5 \mathrm{~cm} \\
11.0\end{array}$ & $\begin{array}{l}1250 \mathrm{gr} \\
710\end{array}$ & 7 \\
\hline 20 & $\mathrm{EZ}$ & $\begin{array}{r}1459 \\
1460 \\
\end{array}$ & જ & $\begin{array}{l}8.0 \\
8.0\end{array}$ & $\begin{array}{r}440 \\
460 \\
\end{array}$ & 6 \\
\hline 21 & $\mathrm{EZ}$ & $\begin{array}{r}1461 \\
1462 \\
\end{array}$ & 우 & $\begin{array}{l}8.0 \\
7.4 \\
\end{array}$ & Unklar & 7 \\
\hline 22 & $\mathrm{EZ}$ & $\begin{array}{r}1467 \\
1468 \\
\end{array}$ & $\delta$ & $\begin{array}{l}10.0 \\
10.5 \\
\end{array}$ & $\begin{array}{l}1235 \\
1240 \\
\end{array}$ & 7 \\
\hline 23 & $\mathrm{EZ}$ & $\begin{array}{l}1469 \\
1470\end{array}$ & $\boldsymbol{\delta}$ & $\begin{array}{l}14.5 \\
14.5 \\
\end{array}$ & $\begin{array}{l}2230 \\
2270 \\
\end{array}$ & 9 \\
\hline 24 & $\mathrm{EZ}$ & $\begin{array}{r}1475 \\
1476 \\
\end{array}$ & $\boldsymbol{\delta}$ & $\begin{array}{r}12.5 \\
\times 2.2 \\
\end{array}$ & $\begin{array}{l}1350 \\
1140 \\
\end{array}$ & 7 \\
\hline 25 & $\mathrm{EZ}$ & $\begin{array}{r}1477 \\
1478 \\
\end{array}$ & 今 & $\begin{array}{l}7.0 \\
7.5 \\
\end{array}$ & $\begin{array}{l}300 \\
200 \\
\end{array}$ & 5 \\
\hline 26 & $\mathrm{EZ}$ & $\begin{array}{l}1514 \\
1515 \\
\end{array}$ & 今 & $\begin{array}{l}13.5 \\
13.5 \\
\end{array}$ & $\begin{array}{l}1475 \\
1290 \\
\end{array}$ & 8 \\
\hline 27 & $\mathrm{EZ}$ & $\begin{array}{l}1520 \\
1521\end{array}$ & 今 & $\begin{array}{l}13.0 \\
13.0\end{array}$ & $\begin{array}{r}2450 \\
2600 \\
\end{array}$ & 10 \\
\hline 28 & $\mathrm{ZZ}$ & $\begin{array}{l}762 \\
763\end{array}$ & $\begin{array}{l}q \\
8\end{array}$ & $\begin{array}{r}12.0 \\
\times 2.0 \\
\end{array}$ & $\begin{array}{r}1020 \\
940\end{array}$ & 8 \\
\hline 29 & $\mathrm{ZZ}$ & $\begin{array}{r}765 \\
769\end{array}$ & $\begin{array}{l}\hat{\delta} \\
+\end{array}$ & $\begin{array}{l}16.2 \\
18.1 \\
\end{array}$ & $\begin{array}{l}2200 \\
2600\end{array}$ & 9 \\
\hline 30 & $\mathrm{ZZ}$ & $\begin{array}{l}954 \\
955\end{array}$ & $\begin{array}{l}\text { q } \\
\text { q }\end{array}$ & $\begin{array}{l}10.5 \\
10.0 \\
\end{array}$ & $\begin{array}{l}465 \\
470 \\
\end{array}$ & 6 \\
\hline $3 I$ & $\mathrm{ZZ}$ & $\begin{array}{l}961 \\
962\end{array}$ & $\begin{array}{l}\hat{\delta} \\
\hat{0}\end{array}$ & $\begin{array}{r}13.0 \\
13.0 \\
\end{array}$ & $\begin{array}{r}1125 \\
980 \\
\end{array}$ & 7 \\
\hline 32 & $\mathrm{ZZ}$ & $\begin{array}{l}1080 \\
1081 \\
\end{array}$ & $\begin{array}{l}\hat{\delta} \\
\text { 우 }\end{array}$ & $\begin{array}{r}14.0 \\
14.5 \\
\end{array}$ & $\begin{array}{l}1235 \\
1140 \\
\end{array}$ & 7 \\
\hline 33 & $\mathrm{ZZ}$ & $\begin{array}{l}1165 \\
1166\end{array}$ & $\begin{array}{l}\hat{\delta} \\
\delta\end{array}$ & $\begin{array}{r}12.5 \\
13.0 \\
\end{array}$ & $\begin{array}{l}1655 \\
1625\end{array}$ & 8 \\
\hline 34 & $Z Z$ & $\begin{array}{r}1323 \\
1324\end{array}$ & 条 & $\begin{array}{l}13.3 \\
15.0 \\
\end{array}$ & $\begin{array}{r}1430 \\
1200 \\
\end{array}$ & 9 \\
\hline 35 & $\overline{Z Z}$ & $\begin{array}{r}1349 \\
1350 \\
\end{array}$ & $\begin{array}{l}\hat{\delta} \\
\text { \& }\end{array}$ & $\begin{array}{l}7.3 \\
6.6 \\
\end{array}$ & $\begin{array}{l}190 \\
180 \\
\end{array}$ & 5 \\
\hline 36 & $\mathrm{ZZ}$ & $\begin{array}{l}1426 \\
1427 \\
\end{array}$ & 후 & $\begin{array}{r}5.7 \\
5.0 \\
\end{array}$ & Unklar & 4 \\
\hline 37 & $\mathrm{ZZ}$ & $\begin{array}{l}1450 \\
\text { I } 45 \mathrm{I}\end{array}$ & 우 & $\begin{array}{l}12.7 \\
12.5\end{array}$ & $\begin{array}{l}1275 \\
1165 \\
\end{array}$ & 7 \\
\hline 38 & $\mathrm{ZZ}$ & $\begin{array}{r}1463 \\
1464 \\
\end{array}$ & $\begin{array}{l}\hat{\delta} \\
\hat{\delta}\end{array}$ & $\begin{array}{l}11.2 \\
11.0\end{array}$ & $\begin{array}{l}1100 \\
1060\end{array}$ & 7 \\
\hline 39 & $Z Z$ & $\begin{array}{l}1465 \\
1466\end{array}$ & $\begin{array}{l}\text { 우 } \\
\text { q }\end{array}$ & $\begin{array}{l}9.6 \\
9.3\end{array}$ & $\begin{array}{l}520 \\
430\end{array}$ & 7 \\
\hline 40 & $\mathrm{ZZ}$ & $\begin{array}{l}1471 \\
1472\end{array}$ & 우 & $\begin{array}{r}13.0 \\
12.5\end{array}$ & $\begin{array}{l}1820 \\
1590\end{array}$ & 10 \\
\hline $4 \mathrm{I}$ & $\mathrm{ZZ}$ & $\begin{array}{r}1473 \\
1474 \\
\end{array}$ & $\begin{array}{l}q \\
+\end{array}$ & $\begin{array}{r}9.2 \\
10.3 \\
\end{array}$ & $\begin{array}{l}580 \\
500\end{array}$ & 6 \\
\hline 42 & Unklar & $\begin{array}{l}540 \\
541\end{array}$ & $\begin{array}{l}\hat{0} \\
\hat{0}\end{array}$ & $\begin{array}{l}10.6 \\
10.4\end{array}$ & $\begin{array}{l}820 \\
750\end{array}$ & 7 \\
\hline 43 & Unklar & $\begin{array}{l}571 \\
572\end{array}$ & $\begin{array}{l}\hat{\delta} \\
\hat{\delta} \\
\end{array}$ & $\begin{array}{l}9.4 \\
8.4\end{array}$ & $\begin{array}{l}600 \\
430\end{array}$ & 7 \\
\hline 44 & Unklar & $\begin{array}{l}1003 \\
1004\end{array}$ & ิे & $\begin{array}{l}11.0 \\
10.5\end{array}$ & $\begin{array}{l}740 \\
645\end{array}$ & 7 \\
\hline 45 & Unklar & $\begin{array}{l}\text { I } 105 \text { (I) } \\
\text { IIOS (II) }\end{array}$ & $\begin{array}{l}\hat{\delta} \\
\hat{\delta}\end{array}$ & Unklar & $\begin{array}{l}1875 \\
1650 \\
\end{array}$ & 10 \\
\hline
\end{tabular}




\section{Eigene Befunde.}

Als sensible Nerven auf der Wade des Menschen sind diejenigen zweierlei Ursprunges zu benennen, d.h. N. cutaneus surae medialis von N. tibialis und $\mathrm{N}$. cutaneus surae lateralis von $\mathrm{N}$. peronaeus communis.

Der N. cutaneus surae medialis entspringt verschieden hoch in der Kniekehle, steigt neben der V. saphena parva in der Rinne zwischen den beiden Bäuchen des M. gastrocunemius abwärts. Er durchbohrt die Fascia cruris etwa in der Mitte des Unterschenkels und zieht subkutan nach lateral abweichend bis hinter den äusseren Knöchel, um als N. suralis und dann N. cutaneus dorsalis pedis lateralis den lateralen Fussrand oder einige Zehen $\mathrm{zu}$ versorgen, wobei er, ausser den Rami calcanei laterales, so gut wie keine Nebenzweige abzusenden pflegt. Der N. cutaneus surae lateralis entspringt in dem oberen lateralen Teile der Kniekehle, teilt sich dann in zwei Äste auf der Rückfläche des Caput laterale $\mathrm{m}$. gastrocunemius. Davon verteilt sich der eine vordere Ast am oberen lateralen Teil der Wade, während der andere hintere Ast, oder der Ramus anastomoticus peronaeus, die Faszia verschieden hoch durchbohrt und nach medial sich begibt, um mit dem N. cutaneus surae medialis zum $\mathrm{N}$. suralis zu anastomosieren. Bei den Feten ist der vordere Ast, meiner Beobachtung nach, in der Mehrzahl der Fälle so schwach ausgebildet, dass man den hinteren Ast als die Hauptfortsetzung des N. cutaneus surae lateralis betrachten kann.

Beim Vergleich der Nervenverteilung in der vorliegenden Untersuchung habe ich die folgenden drei Merkmale ausgewählt :

I. Das Auftreten der Anastomose zwischen den beiden Wadennerven.

2. Die Höhe der Anastomose der beiden Wadennerven.

3. Die Dicke der beiden Wadennerven.

Fall I, EZ, 7 Monate.

I. Fetus, Nr. I68 (I), ô.

Links. Der N. cutaneus surae lateralis ist stärker ausgebildet; er sendet einen schwachen Ast unterhalb der Kniekehle nach medial ab, der mit dem schwächeren $\mathrm{N}$. cutaneus surae medialis im mittleren Drittel des Unterschenkels sich vereinigend, wieder mit dem erstgenannten Nerven anastomosiert.

Rechts. Der N. cut. surae med. ist stärker ausgebildet und vertritt den N. suralis; der schwächere N. cut. surae lat. läuft abwärts bis auf das untere Drittel des Unterschenkels, ohne mit dem N. cut. surae med. zu anastomosieren.

II. Fetus, Nr. I68 (II), 合.

Beiderseits. Der N. cut. surae med. ist stärker ausgebildet und vertritt 
den N. suralis; der N. cut. surae lat. ist dagegen sehr schwach und kurz, indem er nur das obere Drittel des Unterschenkels versorgt.

Vergleich. Am linken Beine des I. Fetus stellt der N. cut. surae lat. den Hauptbestandteil des N. suralis dar, wobei eine mit einer Schlingenbildung von einem Ast des N. cut. surae lat. kombinierende Anastomose zwischen den beiden Wadennerven stattfindet. Die übrigen drei Beine sind darin konkordant, dass der stärkere N. cut. surae med. den N. suralis vertritt, ohne mit dem N. cut. surae lat. zu anastomosieren.

Fall 2, EZ, 7 Monate.

1. Fetus, Nr. $228(\mathrm{I})$, 今.

Links. Die beiden Wadennerven anastomosieren miteinander im mittleren Drittel des Unterschenkels, wobei der N. cut. surae lat. dicker als der N. cut. surae med. ist.

Rechts. Die beiden Wadennerven anastomisieren miteinander im unteren Drittel des Unterschenkels, wobei der N. cut. surae lat. dicker als der N. cut. surae med. ist.

II. Fetus, Nr. 228 (II), 今.

Links. Die beiden Wadennerven anastomosieren miteinander im mittleren Drittel des Unterschenkels, wobei der N. cut. surae lat. dicker als der $\mathrm{N}$, cut. surae med. ist.

Rechts. Die beiden Wadennerven anastomosieren miteinander im unteren Drittel des Unterschenkels, wobei der N. cut. surae lat. dicker als der N. cut. surae med. ist.

Vergleich. Die vier Beine weisen eine Konkordanz untereinander auf hinsichtlich des Auftretens der Anastomose sowie des Dickenverhältnisses der beiden Wadennerven. Über die Höhe der Anastomose findet man eine Konkordanz je nach der Körperseite der beiden Paarlinge.

Fall 3, EZ, 6 Monate.

I. Fetus, Nr. 322, 우.

Links. Die beiden Wadennerven laufen miteinander konvergierend abwärts, bis sie unterhalb des lateralen Knöchels sich vereinigen. Der N. cut. surae med. ist dicker als der $\mathrm{N}$. cut. surae lat.

Rechts. Der N. cut. surae med. vertritt den N. suralis. Der N. cut. surae lat. versorgt, einige schwächere Äste abgebend, die obere Hälfte des Unterschenkels, ohne mit dem $\mathrm{N}$. cut. surae med. zu anastomosieren.

II. Fetus, Nr. 323, 우.

Links. Die beiden Wadennerven anastomosieren miteinander im mittleren Drittel des Unterschenkels, wobei der N. cut. surae med. dicker als der N. cut. surae lat. ist.

Rechts. Der N. cut. surae med. vertritt den N. suralis. Der N. cut. 
surae lat. stellt einen schwächeren Ast auf der oberen Hälfte des Unterschenkels dar, ohne mit dem N. cut. surae med. zu anastomosieren.

Vergleich. Über das Dickenverhältnis der beiden Wadennerven zeigen die vier Beine eine Konkordanz. Über das Auftreten der Anastomose der beiden Wadennerven findet sich nur eine einseitige (Links-links-) Konkordanz, obwohl deren Höhe am linken Beine beider Paarlinge ganz verschieden ist.

Fall 4, EZ, 6 Monate.

I. Fetus, Nr. 399, ô.

Beiderseits. Die beiden Wadennerven anastomosieren miteinander im mittleren Drittel des Unterschenkels. Der N. cut. surae med. ist etwas dicker als der N. cut. surae lat. am linken Beine, während am rechten die beiden Nerven gleichmässig gebildet sind.

II. Fetus, Nr. 400, 今.

Beiderseits. Die beiden Wadennerven anastomosieren miteinander im mittleren Drittel des Unterschenkels. Der N. cut. surae med. ist etwas dicker als der N. cut. surae lat. am rechten Beine, während am linken die beiden Nerven gleichmässig gebildet sind.

Vergleich. Über das Auftreten und die Höhe der Anastomose der beiden Wadennerven findet man eine Konkordanz an den vier Beinen. Über das Dickenverhältnis der beiden Wadennerven bemerkt man jedoch eine stärkere spiegelbildliche Konkordanz.

Fall s, EZ, 9 Monate.

I. Fetus, Nr. s66, ô.

Links. Die beiden Wadennerven anastomosieren miteinander im unteren Drittel des Unterschenkels, wobei der N. cut. surae lat. dicker als der N. cut. surae med. ist.

Rechts. Die beiden Wadennerven anastomosieren miteinander im mittleren Drittel des Unterschenkels, wobei der $\mathrm{N}$. cut. surae lat. dicker als der $N$. cut. surae med. ist.

II. Fetus, Nr. 567, 今.

Links. Die beiden Wadennerven anastomosieren miteinander im unteren Drittel des Unterschenkels, wobei der N. cut. surae lat. dicker als der N. cut. surae med. ist.

Rechts. Die beiden Wadennerven anastomosieren miteinander im mittleren Drittel des Unterschenkels, wobei der N. cut. surae lat. dicker als der $\mathrm{N}$. cut. surae med. ist.

Vergleich. Über das Auftreten der Anastomose und das Dickenverhältnis liegt eine Konkordanz an vier Beinen vor. Über die Höhe der Anastomose findet man eine Links-links- und Rechts-rechts-Konkordanz.

Fall 6, EZ, 7 Monate. 
I. Fetus, Nr. 734 , 9 .

Links. Die beiden Wadennerven anastomosieren miteinander im mittleren Drittel des Unterschenkels, wobei der N. cut. surae lat. dicker als der i. cut. surae med. ist.

Rechts. Der N. cut. surae med. vertritt den N. suralis ; er hat eine kleine Schlinge der Nervenfaser an seinem Wurzelteil. Der N. cut. surae lat. ist schwächer gebildet; er gelangt, feinere Ästchen abgebend, auf das mittlere Drittel des Unterschenkels.

II. Fetus, Nr. 735 , ㅇ.

Links. Die beiden Wadennerven anastomosieren miteinander im oberen Drittel des Unterschenkels (ausserhalb der Kniekehle), wobei der N. cut. surae lat. dicker als der $\mathrm{N}$. cut. surae med. ist.

Rechts. Der N. cut. surae med. vertritt den N. suralis, während der N. cut. surae lat. die untere Hälfte des Unterschenkels kaum erreicht, ohne mit dem N. cut. surae med. zu anastomosieren.

Vergleich. Über das Vorkommen der Anastomose und das Dickenverhältnis der beiden Wadennerven findet sich nur eine Links-links-Konkordanz, wenngleich die Höhe der Anastomose der Nerven an diesen beiden Beinen nicht gleich ist.

Fall 7, EZ, 8 Monate.

I. Fetus, Nr. 768 , 우.

Links. Die beiden Wadennerven anastomosieren miteinander im mittleren Drittel des Unterschenkels, wobei der einige schwache Äste nach unten lateral abgebende $\mathrm{N}$. cut. surae lat. schwächer als der $\mathrm{N}$. cut. surae med. ist.

Rechts. Die beiden gleich dicken Wadennerven anastomosieren miteinander im mittleren Drittel des Unterschenkels. Ein stärkerer Ast entspringt aus dem Stamm des N. cut. surae lat., der über den N. cut. surae med. hinüberkreuzend, bis auf die Höhe des Malleolus sich fortsetzt, ohne mit dem N. suralis zu anastomosieren.

II. Fetus, Nr. 770 , 우.

Links. Die beiden Wadennerven anastomosieren miteinander im unteren Drittel des Unterschenkels, wobei der einen schwachen Ast nach unten lateral abgebende N. cut. surae lat. schwächer als der N. cut. surae med. ist.

Rechts. Die beiden Wadennerven anastomosieren miteinander im unteren Drittel des Unterschenkels. Der dickere N. cut. surae lat. sendet einen schwachen Ast von dem Stamm nach medialwärts ab, der bald sich gabelt, um einen schmalen Hautteil zwischen den beiden Wadennerven zu versorgen.

Vergleich. Die vier Beine sind nur darin konkordant, dass die Anastomose der beiden W'adennerven stattfindet, obwohl dabei die Höhe der Anastomose bei beiden Paarlingen etwas verschieden ist. Über das Dickenverhältnis 
liegt nur eine Links-links-Konkordanz vor.

Fall 8, EZ, s Monate.

I. Fetus, Nr. 825 , 우.

Links. Der stärkere N. cut. surae lat. zweigt sich von der Teilungsstelle des $\mathrm{N}$. peronaeus communis und $\mathrm{N}$. tibialis, also dicht nahe dem Stamm des schwächeren $N$. cut. surae med. ab. Die beiden Wadennerven steigen miteinander sich fast berührend abwärts, um sich im unteren Drittel des Unterschenkels zu vereinigen.

Rechts. Hier findet man eine Varietät der Nervenversorgung. Der N. cutaneus femoris posterior setzt sich auf den Wadenteil fort, um sich an der sensiblen Innervation dort zu beteiligen, indem er mit einem stärkeren Ast aus dem N. tibialis (N. cut. surae med.) im unteren Drittel des Unterschenkels anastomosiert. Es fehlt hier zwar der Ast aus dem N. peronaeus communis (N. cut. surae lat.).

II. Fetus, Nr. 826, 우.

Links. Die beiden Wadennerven anastomosieren miteinander im unteren Drittel des Unterschenkels, wobei der $\mathrm{N}$. cut. surae lat. dicker als der $\mathrm{N}$. cut. surae med. ist.

Rechts. Die Teilungsstelle der beiden Wadennerven aus dem Stammnerven näheren sich als gewöhnlich. Die beiden Nerven anastomosieren miteinander im unteren Drittel des Unterschenkels, wobei der N. cut. surae lat. dicker als der $\mathrm{N}$. cut. surae med. ist.

Vergleich. Unter drei Beinen besteht eine Konkordanz über die Höhe der Anastomose und das Dickenverhältnis der beiden Wadennerven. Bezüglich des Verhaltens der beiden Nerven an ihren Wurzel weisen die zwei Beine eine auffallende spiegelbildliche Konkordanz auf.

An der rechten Wade des I. Paarlinges findet man eine seltene Varietät der Nervenversorgung, die durch die Fortsetzung des N. cut. femoris posterior und das Fehlen des N. cut. surae lat. ausgezeichnet ist. Diese Varietät ist schon von Kosinski (1926) und Ushio (1934) beschrieben worden. $\mathrm{K}$ os in ski gibt an, diese vergleichend-anatomisch primitive Form der Nervenverteilung an den beiden Beinen eines Erwachsenen (in $1.7 \%$ der Fälle) gefunden zu haben. Ushio hat diese Varietät bei 4 unter 20 Fällen beobachtet, bei denen der N. cut. fem. post. mit dem N. cut. surae lat. in Verbindung steht, die aber sich von meiner dadurch unterscheidet, dass die Anastomose zwischen dem N. cut. fem. post. und dem N. cut. surae med. stattfindet.

Fall 9, E'Z, 7 Monate.

I. Fetus, Nr. IоI2, 우.

Links. Die beiden Wadennerven anastomosieren miteinander im unteren Drittel des Unterschenkels, wobei der N. cut. surae lat., der den vorderen 
Ast nach lateral unten absendet, dicker als der N. cut. surae med. ist.

Rechts. Die beidęn Wadennerven anastomosieren miteinander im mittleren Drittel des Unterschenkels, wobei der N. cut. surae lat., der den vorderen Ast nach lateral unten abgibt, dicker als der N. cut. surae med. ist.

II. Fetus, Nr. IOI3, 우.

Beiderseits. Die beiden Wadennerven anastomosieren miteinander im mittleren Drittel des Unterschenkels, wobei der N. cut. surae lat., der den vorderen Ast nach lateral unten absendet, dicker als der N. cut. surae med. ist.

Vergleich. Über das Auftreten der Anastomose und das Dickenverhältnis der beiden Wadennerven sind alle vier Beine konkordant. Ausgenommen vom linken Beine des I. Paarlings, sind die drei Beine dieses Paares auch konkordant über die Höhe der Anastomose.

Fall 10, EZ, 6 Monate.

I. Fetus, Nr. 1063 , 우.

Links. Die beiden Wadennerven anastomosieren miteinander im mittleren Drittel des Unterschenkels, wobei der N. cut. surae lat. dicker als der N. cut. surae med. ist.

Rechts. Der starke N. cut. surae med. vertritt den N. suralis, wobei das Versorgungsgebiet des N. cut. surae lat. nur auf dem lateralen Kopf des Wadenmuskels reduziert ist.

II. Fetus, Nr. I064, 우.

Links. Der stärkere N. cut. surae med. vertritt den N. suralis. Der N. cut. surae lat. ist schwächer; er gelangt, einige Äste nach lateral abgebend, auf das untere Drittel des Unterschenkels, ohne mit dem N. cut. surae med. $z u$ anastomosieren.

Vergleich. Über das Auftreten der Anastomose und das Dickenverhältnis der beiden Wadennerven besteht eine spiegelbildliche Konkordanz. Über die Höhe der Anastomose weist je ein Bein der beiden Paarlinge in spiegelbildlicher Richtung eine Konkordanz untereinander auf.

Fall I I, EZ, 9 Monate.

I. Fetus, Nr. I I07, 令.

Beiderseits. Die Teilungsstellen der beiden Wadennerven von dem Stammnerven näheren sich miteinander so dicht, dass sie von einer" gemeinsamen Wurzel entspringen. Die beiden Wadennerven anastomosieren miteinander im mittleren Drittel des Unterschenkels, obwohl die Vereinigungsstelle am rechten Beine etwas höher als die am linken ist. Der N. cut. surae lat. hat einen stärkeren vorderen Ast, der etwa die Mitte des Unterschenkels erreicht.

II. Fetus, Nr. ı 108 , 今.

Links. Die Teilungsstellen der beiden Wadennerven aus dem Stamm- 
nerven näheren sich als gewöhnlich miteinander. Die beiden Nerven anastomosieren miteinander im unteren Drittel des Unterschenkels. Der N. cut. surae lat. ist dicker als der N. cut. surae med. Unterhalb der Kniekehle senden die beiden Nerven je einen Ast ab, der voneinander divergierend, sich bis auf das mittlere Drittel des Unterschenkels fortsetzt.

Rechts. Die Teilungsstellen der beiden Wadennerven aus dem Stammnerven näheren sich miteinander so dicht, dass sie von einer gemeinsamen Wurzel entspringen. Der $N$. cut. surae lat. gabelt sich hart nahe seinem Ursprung; der mediale Schenkel davon anastomosiert mit dem N. cut. surae med. im oberen Drittel (ausserhalb der Kniekehle), während der laterale, feinere Äste nach beiderseits abgebend, auf den oberen Teil des lateralen Knöchels gelangt.

Vergleich. Über das Auftreten der Anastomose und das Dickenverhältnis der beiden Wadennerven sind die vier Beine konkordant, obwohl die Höhe der Anastomose nur beim I. Paarling beiderseits ähnlich ist.

Fall i 2, EZ, 8 Monate.

I. Fetus, Nr. I 254 , ô.

Beiderseits. Der N. cut. surae med. ist stärker ausgebildet und vertritt den N. suralis, indem das Versorgungsgebiet des N. cut. surae lat. auf einem schmalen Hautteil auf dem lateralen Kopf des Wadenmuskels beschränkt ist.

II. Fetus, Nr. I255, 今.

Beiderseits. Der N. cut. surae med. ist stärker ausgebildet und vertritt den $\mathrm{N}$. suralis, indem das Versorgungsgebiet des N. cut. surae lat. auf einem schmalen Hautteil auf dem lateralen Kopf des Wadenmuskels beschränkt ist.

Vergleich. Die vier Beine sind darin recht konkordant, dass ihnen eine Anastomose der beiden Wadennerven fehlt und der starke N. cut. surae med. den $N$. suralis vertritt.

Fall I 3, EZ, 7 Monate.

I. Fetus, Nr. i26r, 우.

Links. Der N. peronaeus communis liefert zwei Äste zum Wadenteil ; der eine stärkere Ast tritt, mit einem schwachen Schenkel von dem N. tibialis innerhalb der Kniekehle vereinigend, auf den Wadenteil hervor, um den $\mathrm{N}$. suralis zu vertreten. Der andere schwächere Ast läuft, mehrere feine Äste nach beiderseits abgebend, bis zum unteren Drittel des Unterschenkels abwärts.

Rechts. Die beiden gleich dicken Wadennerven anastomosieren miteinander im mittleren Drittel des Unterschenkels, um den N. suralis auszubilden.

II. Fetus, Nr. I262, 우.

Links. Der N. cut. surae lat. ist stark ausgebildet ; er sendet nach beiderseits je einen schwachen Ast ab, und anastomosiert mit dem sehr feinen N. cut. surae med. im mittleren Drittel des Unterschenkels. 
Rechts. Die beiden gleich dicken Wadennerven anastomosieren miteinander im mittleren Drittel des Unterschenkels. Der N. cut. surae lat. sendet den rorderen Ast nach lateral unten ab.

Vergleich. Über das Auftreten der Anastomose der beiden Wadennerven sind die vier Beine und über die Höhe der Anastomose drei Beine konkordant. Das Dickenverhältnis ist in der gleichnamigen Körperseite bei beiden Paarlingen konkordant.

Fall 14, EZ, 9 Monate.

I. Fetus, Nr. I300, 合.

Beiderseits. Die beiden Wadennerven anastomosieren miteinander im mittleren Drittel des Unterschenkels, wobei der $\mathrm{N}$. cut. surae lat. auffallend dicker als der $N$. cut. surae med. ist.

II. Fetus, Nr. Izor, ô.

Links. Der N. cut. surae lat. teilt sich unterhalb der Kniekehle in zwei gleichmässige Äste; davon empfängt der medialwärts laufende Ast den schwachen $\lambda$. cut. surae med.; er vereinigt sich wieder mit dem lateralwärts laufenden Ast im mittleren Drittel des Unterschenkels. Hier entsteht daher eine dickere Schlinge von dem N. cut. surae lat.

Rechts. Die beiden Wadennerven anastomosieren miteinander im mittleren Drittel des Unterschenkels, wobei der ein schwaches Ästchen abgebende $\mathrm{N}$. cut. surae lat. dicker als der N. cut. surae med. ist.

Vergleich. Hinsichtlich der drei Merkmale, der Anastomose, deren Höhe und des Dickenverhältnis, sind die vier Beine konkordant, wenngleich das linke Bein des II. Fetus durch eine Schlingenbildung der Nerven ausgezeichnet ist.

Fall is, EZ, 7 Monate.

I. Fetus, Nr. I424, 今.

Beiderseits. Die beiden gleich dicken Wadennerven anastomosieren miteinander im mittleren Drittel des Unterschenkels. Der N. cut. surae lat. sendet einen Ast nach unten bis auf die Mitte des Wadenteils ab.

II. Fetus, Nr. 1425 , ô.

Links. Die beiden gleich dicken Wadennerven laufen miteinander konvergierend abwärts und vereinigen sich erst hinter dem äusseren Knöchel.

Rechts. Die beiden gleich dicken Wadennerven anastomosieren miteinander im oberen Drittel (ausserhalb der Kniekehle) des Unterschenkels.

Vergleich. Über das Auftreten der Anastomose und das Dickenverhältnis der beiden Wadennerven sind die vier Beine konkordant. Hinsichtlich der Höhe der Anastomose ist die symmetrische Verteilung nur beim I. Paarling zu sehen, von der beim II. Paarling keineswegs die Rede ist.

Fall 16, EZ, 7 Monate. 
I. Fetus, Nr. I43I, 우.

Beiderseits. Die beiden gleich dicken Wadennerven laufen miteinander konvergierend abwärts, bis sie im unteren Drittel des Unterschenkels sich vereinigen. Der N. cut. surae lat. trägt einen schwachen vorderen Ast.

II. Fetus, Nr. 1432, ㅇ․

Beiderseits. Die beiden gleich dicken Wadennerven laufen miteinander konvergierend abwärts, bis sie erst hinter dem äusseren Knöchel sich vereinigen.

Vergleich. Die vier Beine sind sehr konkordant bezüglich der gleichen Mächtigkeit und der niedrigen Vereinigungsstelle der beiden Wadennerven, obwohl die letztere beim I. Paarling etwas höher als beim II. ist.

Fall 17, EZ, 9 Monate.

I. Fetus, Nr. 1445, 今.

Beiderseits. Der $\mathrm{N}$. cut. surae med. ist stärker ausgebildet und vertritt den N. suralis, wobei der N. cut. surae lat. mehrere feine Äste nach beiderseits abgebend, bis auf das untere Drittel des Unterschenkels sich fortsetzt, ohne mit dem N. cut. surae med. zu anastomosieren.

II. Fetus, Nr. 1446, 今.

Beiderseits. Der N. cut. surae med. ist stärker ausgebildet und vertritt den N. suralis, wobei der N. cut. surae lat. mehrere feine Äste nach beiderseits abgebend, bis auf das mittlere Drittel des Unterschenkels sich fortsetzt, ohne mit dem $\mathrm{N}$. cut. surae med. zu anastomosieren.

Vergleich. Man findet eine ausgesprochene Konkordanz der Nervenverteilung unter den vier Beinen, d.h. der stark ausgebildete $N$. cut. surae med. allein vertritt den $N$. suralis.

Fall i 8, EZ, 8 Monate.

I. Fetus, Nr. 1453, 今.

Links. Der N. cut. surae med. empfängt schon in der Kniekehle einen kurzen Anastomosenast von dem N. peronaeus communis. Ein schwacher Ast zweigt sich ausserdem von der Wurzel des peronaealen Schenkels, der nur die laterale obere Hälfte des Wadenteils versorgt.

Rechts. Der stark ausgebildete $N$. cut. surae lat. gabelt sich an seinem Stamm ; davon vertritt der eine stärkere medial befindliche Ast den N. suralis, ohne mit dem schwachen $N$. cut. surae med. zu anastomosieren, der andere schwächere Ast erreicht das untere Drittel des Unterschenkels.

II. Fetus, Nr. 1454, 今.

Beiderseits. Die beiden Wadennerven anastomosieren miteinander im mittleren Drittel des Unterschenkels, wobei der N. cut. surae lat. dicker als der N. cut. surae med. ist. Am rechten Beine befindet sich ein schwacher Ast peronaealen Ursprunges zwischen den beiden Wadennerven. 
Vergleich. Über die Befunde der beiden Wadennerven sind die beiden Paarlinge stark diskordant. Beim I. Paarling geht zwar die Verteilung nach der Körperseite stark auseinander, wenngleich sie beim II. symmetrisch ähnlich ist.

Fall $19, \mathrm{EZ}, 7$ Monate.

I. Fetus, Nr. 1457, ㅇ.

Links. Der N. cut. surae lat. ist stärker gebildet und vertritt den N. suralis. Der N. cut. surae med. läuft auf der Mittellinie des Wadenteils, ohne mit dem N. suralis zu anastomosieren.

Rechts. Die beiden Wadennerven laufen miteinander konvergierend abwärts, bis sie im unteren Drittel des Unterschenkels sich vereinigen, wobei der N. cut. surae lat. dicker als der N. cut. surae med. ist.

II. Fetus, Nr. 1458 , 우.

Links. Der N. cut. surae lat. ist stärker gebildet und vertritt den N. suralis. Der N. cut. surae med. läuft auf der Mittellinie des Wadenteils, ohne mit dem N. suralis zu anastomosieren.

Rechts. Die beiden Wadennerven laufen miteinander konvergierend abwärts, bis sie im unteren Drittel des Unterschenkels sich vereinigen, wobei der N. cut. surae lat. dicker als der N. cut. surae med. ist.

Vergleich. Über das Dickenverhältnis der beiden Wadennerven zeigen die vier Beine eine Konkordanz. Über das Vorkommen und die Höhe der Anastomose findet man eine merkwürdige einseitige Konkordanz.

Fall 20, EZ, 6 Monate.

I. Fetus, Nr. 1459 , ô.

Links. Die beiden Wadennerven anastomosieren miteinander im mittleren Drittel des Unterschenkels, wobei der N. cut. surae lat., der den feinen vorderen Ast von seinem Stamm nach lateral unten abgibt, dicker als der N. cut. surae med. ist.

Rechts. Der N. cut. surae med. ist stärker gebildet und vertritt den N. suralis. Der schwache N. cut. surae lat. gelangt, einige feine Ästchen nach beiderseits abgebend, auf das untere Drittel des Unterschenkels, ohne mit dem $N$. cut. surae med. zu anastomosieren.

II. Fetus, Nr. 1460 , $\hat{\delta}$.

Links. Die beiden Wadennerven anastomosieren miteinander im mittleren Drittel des Unterschenkels, wobei der N. cut. surae lat., der den feinen vorderen Ast von seinem Stamm nach lateral unten abgibt, dicker als der N. cut. surae med. ist.

Rechts. Der N. cut. surae med. ist stärker gebildet und vertritt den N. suralis. Der schwache N. cut. surae lat. gelangt, einige feine Ästchen abgebend, auf das untere Drittel des Unterschenkels, ohne mit dem N. cut. 
surae med. zu anastomosieren.

Vergleich. Über das Auftreten sowie die Höhe der Anastomose und das Dickenverhältnis der beiden Wadennerven befindet sich eine interessante jederseitige Konkordanz.

Fall 21, EZ, 7 Monate.

I. Fetus, Nr. I46I, 우.

Links. Der N. cut. surae lat. gabelt sich kurz nach dem Abzweigen von dem N. peronaeus communis; davon erreicht der eine lateral befindliche Ast das mittlere Drittel des Unterschenkels, während der andere medial befindliche mit dem $N$. cut. surae med. von gleicher Mächtgkeit miteinander konvergierend absteigt, um endlich unterhalb des äusseren Knöchels sich vereinigen.

Rechts. Die beiden Wadennerven anastomosieren miteinander im unteren Drittel des Unterschenkels, wobei der N. cut. surae lat., der an seiner Wurzel eine langgedehnte Schlinge bildet, dicker als der N. cut. surae med. ist.

II. Fetus, Nr. 1462, 우.

Links. Die beiden Wadennerven anastomosieren miteinander im unteren Drittel des Unterschenkels, wobei der N. cut. surae lat., der an seiner Wurzel eine langgedehnte Schlinge bildet, dicker als der N. cut. surae med. ist.

Rechts. Die beiden Wadennerven anastomosieren miteinander im unteren Drittel des Unterschenkels, wobei der N. 'cut. surae lat., der einen schwachen Ast nach medial abgibt, dicker als der N. cut. surae med. ist. Dicht oberhalb der Vereinigungsstelle der beiden Wadennerven findet man noch eine feine Brücke der Nervenfaser.

Vergleich. Über das Auftreten der Anastomose sind die vier Beine konkordant. Über die Höhe der Anastomose und das Dickenverhältnis der beiden Wadennerven sind drei Beine, ausgenommen von dem linken des I. Paarlinges, konkordant, obwohl dabei, streng genommen, der Modus der Verästelung und der Anastomose der Nerven nicht gleichsinnig ist.

Fall 22, EZ, 7 Monate.

I. Fetus, Nr. 1467 , ô.

Links. Die beiden Wadennerven anastomosieren miteinander im mittleren Drittel des Unterschenkels, wobei der N. cut. surae lat., der zwei Äste in der Höhe des mittleren Drittels nach lateral unten abgibt, dicker als der N. cut. surae med. ist.

Rechts. Die beiden Wadennerven anastomosieren miteinander im unteren Drittel des Unterschenkels, wobei der N. cut. surae lat. dicker als der N. cut. surae med. ist.

II. Fetus, Nr. I468, 令. 
Beiderseits. Die beiden Wadennerven von gleicher Mächtigkeit anastomosieren miteinander im mittleren Drittel des Unterschenkels. Der N. cut. surae lat. sendet einen Ast von seinem Stamm nach medial unten (am linken Beine) oder nach lateral unten (am rechten Beine) ab.

Vergleich. Über das Auftreten der Anastomose der beiden Wadennerven sind vier Beine, und über die Höhe der Anastomose drei Beine konkordant, obwohl die beiden Paarlinge über das Dickenverhältnis der beiden Nerven ganz diskordant sind.

Fall $23, \mathrm{EZ}, 9$ Monate.

I. Fetus, Nr. 1469 , ô.

Beiderseits. Die beiden Wadennerven anastomosieren miteinander im unteren Drittel des Unterschenkels, wobei der N. cut. surae lat., der einen kurzen $A$ st nach medial unten abgibt, dicker als der $N$. cut. surae med. ist.

II. Fetus, Nr. 1470, 冬.

Links. Die beiden Wadennerven anastomosieren miteinander im mittleren Drittel des Unterschenkels, wobei der N. cut. surae med. dicker als der N. cut. surae lat. ist.

Rechts. Der N. cut. surae lat. ist durch die Vereinigung der zwei langen Schenkel aus dem N. peronaeus communis gebildet. Die beiden Wadennerven von gleicher Mächtigkeit anastomosieren miteinander im mittleren Drittel des Unterschenkels.

Vergleich. Über das Auftreten der Anastomose der beiden Wadennerven sind die vier Beine konkordant. Die Höhe der Anastomose ist je nach dem Fetus beiderseits symmetrisch ähnlich, obwohl am rechten Beine des II. Paarlings eine Schlingenbildung an der Wurzel des N. cut. surae lat. zu sehen ist. Über das Dickenverhältnis der beiden Nerven besteht eine Diskordanz zwischen den beiden Paarlingen.

Fall 24, EZ, 7 Monate.

I. Fetus, Nr. 1475 , 命.

Beiderseits. Die beiden Wadennerven anastomosieren miteinander im mittleren Drittel des Unterschenkels, wobei der N. cut. surae lat., der den schwachen vorderen Ast nach lateral unten entsendet, dicker als der $\mathrm{N}$. cut. surae med. ist.

II. Fetus, Nr. 1476 , ô.

Beiderseits. Die beiden Wadennerven anastomosieren miteinander im mittleren Drittel des Unterschenkels, wobei der N. cut. surae lat., der den schwachen vorderen Ast nach lateral unten entsendet, dicker als der $\mathrm{N}$. cut. surae med. ist.

Vergleich. Die vier Beine sind sehr konkordant bezüglich der Nervenverteilung, indem sich der $\mathrm{N}$. cut. surae lat. mit dem schwächeren $\mathrm{N}$. cut. surae 
med. im mittleren Drittel des Unterschenkels vereinigt.

Fall 25, EZ, 5 Monate.

I. Fetus, Nr. 1477, o.

Beiderseits. Die beiden Wadennerven anastomosieren miteinander im mittleren Drittel des Unterschenkels, wobei der N. cut. surae lat., der einen oberflächlichen Ast von seinem Stamm auf das untere Drittel des Unterschenkels entsendet, dicker als der N. cut. surae med. ist.

II. Fetus, Nr. 1478, 今.

Beiderseits. Die beiden Wadennerven anastomosieren miteinander im mittleren Drittel des Unterschenkels, wobei der N. cut. surae lat., der einige feine oberflächliche Äste nach beiderseits entsendet, dicker als der N. cut. surae med. ist.

Vergleich. Die vier Beine sind konkordant bezüglich der Nervenverteilung, indem der $\mathrm{N}$. cut. surae lat. mit dem schwächeren $\mathrm{N}$. cut. surae med. im mittleren Drittel des Unterschenkels anastomosiert. . Der Verlauf des feineren oberflächlichen Astes von dem $N$. cut. surae lat. ist je nach dem Fetus etwas verschieden.

Fall 26, EZ, 8 Monate.

I. Fetus, Nr. I 5 I4, ô.

Beiderseits. Die beiden Wadennerven anastomosieren miteinander im mittleren Drittel des Unterschenkels, wobei der N. cut. surae lat. dicker als der N. cut. surae med. ist. Ein mässig dicker Ast entspringt aus dem N. cut. surae lat. ausserhalb der Kniekehle, der oberflächlich den N. cut. surae med. durchquerend, wieder sich mit dem N. suralis verbindet. Hier bildet sich also eine Nervenschlinge an dem $N$. cut. surae lat. in der oberen Hälfte des Unterschenkels.

II. Fetus, Nr. I I I , $\hat{o}$.

Beiderseits. Der N. peronaeus communis entsendet zwei Äste zum Wadenteil; der eine schwächere lateral befindliche Ast setzt sich, mehrere feine Ästchen nach beiderseits abgebend, bis auf das untere Drittel des Unterschenkels fort, während der andere stärkere medial befindliche den $\mathrm{N}$. cut. surae lat. vertritt. Der letztere gabelt sich bald ausserhalb der Kniekehle und anastomosiert verdoppelt mit dem schwachen $N$. cut. surae med., wobei er einen kleinen Ring von Nervenfaser (am linken Beine etwas höher als am rechten) bildet.

Vergleich. Über das Auftreten der Anastomose, welche durch eine Schlingenbildung des Nerven ausgezeichnet ist, und das Dickenverhältnis der beiden Wadennerven zeigen alle vier Beine eine interessante Konkordanz. Die Verästelungsweise und die Höhe der Anastomose ist je nach dem Fetus symmetrisch ähnlich. 
Fall 27, EZ, io Monate.

I. Fetus, Nr. I 520 , o.

Links. Die Anastomose zwischen den beiden Wadennerven findet verdoppelt im unteren Drittel des Unterschenkels sowie unterhalb des äusseren Knöchels statt, wobei der N. cut. surae lat. dicker als der N. cut. surae med. ist. Ein schwacher oberflächerer Ast zweigt sich von dem Stamm des $N$. cut. surae lat. ab, der den $N$. cut. surae med. durchquerend, auf das mittlere Drittel des Unterschenkels gelangt.

Rechts. Die beiden Wadennerven anastomosieren miteinander erst unterhalb des äusseren Knöchels, wobei der N. cut. surae lat., der den schwachen vorderen $\lambda$ st nach lateral unten entsendet, dicker als der N. cut. surae med. ist.

II. Fetus, Nr. I 52 I, 今.

Links. Die beiden Wadennerven anastomosieren miteinander im unteren Drittel des Lnterschenkels, wobei der N. cut. surae lat., der den schwachen vorderen Ast nach lateral unten absendet, dicker als der N. cut. surae med. ist.

Rechts. Die beiden Wadennerven anastomosieren miteinander im mittleren Drittel des Unterschenkels, wobei der N. cut. surae lat. dicker als der N. cut. surae med. ist.

Vergleich. Über das Auftreten der Anastomose und das Dickenverhältnis der beiden Wadennerven sind die vier Beine konkordant, obwohl sie über die Höhe der Anastomose ganz diskordant sind. Am linken Beine des I. Paarlings bemerkt man eine Doppelanastomose zwischen den beiden Nerven.

Fall 28, ZZ, 8 Monate.

I. Fetus, Nr. 762 , 우.

Links. Die beiden Wadennerven anastomosieren miteinander im mittleren Drittel des Unterschenkels, wobei der N. cut. surae lat., der den vorderen Ast nach lateral unten absendet, dicker als der N. cut. surae med. ist.

Rechts. Die beiden gleich dicken Wadennerven anastomosieren miteinander im mittleren Drittel des Unterschenkels. Der N. cut. surae lat. liefert den vorderen Ast nach lateral unten.

II. Fetus, Nr. $76_{3}$, ô.

Links. Die beiden gleich dicken Wadennerven anastomosieren miteinander im mittleren Drittel des Unterschenkels. Der N. cut. surae lat. liefert den vorderen Ast nach lateral unten.

Rechts. Die beiden Wadennerven anastomosieren miteinander im mittleren Drittel des Unterschenkels, wobei der N. cut. surae lat., der den vorderen Ast nach lateral unten entsendet, dicker als der $\mathrm{N}$. cut. surae med. ist.

Vergleich. Über das Auftreten sowie die Höhe der Anastomose findet sich eine Konkordanz an vier Beinen, obwohl dabei, streng genommen, über 
das Dickenverhältnis und die Höhe der Anastomose der beiden Wadennerven eine ausgesprochene spiegelbildliche Konkordanz besteht.

Fall 29, ZZ, 9 Monate.

I. Fetus, Nr. 765 , 今.

Beiderseits. Die beiden Wadennerven anastomosieren miteinander im mittleren Drittel des Unterschenkels, wobei der N. cut. surae lat., der den vorderen Ast von seinem Stamm zum unteren Drittel des Unterschenkels absendet, dicker als der N. cut. surae med. ist.

II. Fetus, Nr. 769 우.

Links. Die beiden Wadennerven anastomosieren miteinander im unteren Drittel des Unterschenkels, wobei der N. cut. surae lat., der den vorderen Ast nach lateral unten absendet, dicker als der $\mathrm{N}$. cut. surae med. ist.

Rechts. Der stark gebildete N. cut. surae med. vertritt den N. suralis. Der N. cut. surae lat. ist schwach ; er gelangt auf das untere Drittel des Unterschenkels, ohne mit dem N. cut. surae med. zu anastomosieren.

Vergleich. Über das Auftreten der Anastomose sowie das Dickenverhältnis der beiden Wadennerven sind die drei Beine, ausgenommen von dem rechten des II. Paarlings, konkordant. Über die Höhe der Anastomose sind nur die beiden Beine des I. Paarlings symmetrisch ähnlich.

Fall 30, ZZ, 6 Monate.

I. Fetus, Nr. 954, 우.

Links. Die beiden Wadennerven anastomosieren miteinander im unteren Drittel des Unterschenkels, wobei der N. cut. surae lat. dicker als der N. cut. surae med. ist.

Rechts. Die beiden Wadennerven laufen miteinander konvergierend abwärts, ohne sich zu vereinigen. Der stärkere N. cut. surae lat., der den feinen vorderen Ast nach lateral unten entsendet, vertritt den N. suralis.

II. Fetus, Nr. 955, 우.

Links. Die beiden Wadennerven anastomosieren miteinander erst unterhalb des äusseren Knöchels, wobei der N. cut. surae lat. dicker als der N. cut. surae med. ist.

Rechts. Der stark gebildete N. cut. surae med. vertritt den N. suralis. Der N. cut. surae lat. gelangt, einige Ästchen abgebend, auf die Höhe des Knöchels, ohne mit dem N. cut. surae lat. zu anastomosieren.

Vergleich. Über das Dickenverhältnis der beiden Wadennerven sind die drei Beine, d.h.ausgenommen von dem rechten des II. Paarlings, konkordant. Über das Auftreten der Anastomose findet man nur die einseitige (Linkslinks-) Konkordanz, obwohl dabei die Höhe derselben ganz verschieden ist.

Fall 31, ZZ, 7 Monate.

I. Fetus, Nr. 96r, 今. 
Links. Die beiden Wadennerven anastomosieren miteinander im mittleren Drittel des Unterschenkels, wobei der N. cut. surae lat., der den kurzen vorderen Ast nach lateral unten absendet, dicker als der N. cut. surae med. ist.

Rechts. Die beiden Wadennerven laufen miteinander konvergierend abwärts, bis sie hinter dem äusseren Knöchel sich vereinigen. Der N. cut. surae lat., der den kurzen vorderen Ast nach lateral unten absendet, ist dicker als der $\mathrm{N}$. cut. surae med.

II. Fetus, Nr. 962, 今.

Links. Der N. cut. surae lat., der den vorderen Ast von dem Stamm nach lateral unten absendet, bildet einen Nervenring in der oberen Hälfte des Unterschenkels. Er anastomosiert verdoppelt mit dem schwachen N. cut. surae med. in der Höhe des mittleren und unteren Drittels des Unterschenkels.

Rechts. Die beiden Wadennerven anastomosieren miteinander im unteren Drittel des Unterschenkels, wobei der N. cut. surae lat., der einige kurze Äste nach lateral unten absendet, dicker als der N. cut. surae med. ist.

Vergleich. Über das Auftreten der Anastomose und das Dickenverhältnis der beiden Wadennerven sind die vier Beine konkordant, obwohl sie über die Höhe der Anastomose ganz verschieden sind. Am linken Beine des II. Paarlings bemerkt man einen Nervenring und eine Doppelverbindung zwischen den beiden Wadennerven.

Fall 32, ZZ, 7 Monate.

I. Fetus, Nr. I080, $\hat{\text { o. }}$

Beiderseits. Die beiden Wadennerven anastomosieren miteinander im mittleren Drittel des Unterschenkels, wobei der N. cut. surae lat. dicker als der N. cut. surae med. ist.

II. Fetus. Nr. 1081, 우.

Beiderseits. Die beiden Wadennerven anastomosieren miteinander im mittleren Drittel des Unterschenkels, wobei der N. cut. surae lat. dicker als der N. cut. surae med. ist.

Vergleich. Über die Nervenverteilung findet man eine ausgesprochene Konkordanz unter allen vier Beine, an denen der stärkere N. cut. surae lat. mit dem schwächeren N. cut. surae med. im mittleren Drittel des Unterschenkels anastomosiert.

Fall 33, ZZ, 8 Monate.

I. Fetus, Nr. I165, 各.

Links. Die beiden Wadennerven anastomosieren miteinander im unteren Drittel des Unterschenkels, wobei der N. cut. surae med. dicker als der N. cut. surae lat. ist.

Rechts. Die beiden Wadennerven anastomosieren miteinander im unteren Drittel des Unterschenkels, wobei der N. cut. surae lat. dicker als der N. 
cut. surae med. ist.

II. Fetus, Nr. i 166 . ô,

Links. Im mittleren Drittel des Unterschenkels liefert der den N. suralis vertretende stärkere $N$. cut. surae lat. einen feinen Verbindungsast zum schwach gebildeten N. cut. surae med.

Rechts. Die beiden Wadennerven anastomosieren miteinander im mittleren.Drittel des Unterschenkels, wobei der N. cut. surae lat., der den schwächeren vorderen Ast zum oberen Drittel des Unterschenkels absendet, dicker als der N. cut. surae med. ist.

Vergleich. Über das Auftreten der Anastomose der beiden Wadennerven sind die vier Beine konkordant. Über die Höhe der Anastomose findet sich eine Diskordanz zwischen den beiden Paarlingen. Über das Dickenverhältnis sind drei Beine, d.h. ausgenommen von dem linken des I. Paarlings, konkordant.

Fall 34, ZZ, 9 Monate.

I. Fetus, Nr. I323, ô.

Beiderseits. Von der Teilungsstelle des N. tibialis und N. peronaeus communis zweigt sich der $N$. cut. surae lat. ab, der einen kurzen Ast nach lateral unten abgebend, mit dem schwachen $\mathrm{N}$. cut. surae med. im unteren Drittel des Unterschenkels anastomosiert, um den N. suralis zu vertreten.

II. Fetus, Nr. 1324, o.

Links. Von der Teilungsstelle des N. tibialis und N. peronaeus communis zweigt sich der $\mathrm{N}$. cut. surae lat. ab, der einen kurzen Ast nach lateral unten abgebend, mit dem schwachen $\mathrm{N}$. cut. surae med. im unteren Drittel des Unterschenkels anastomosiert, um den N. suralis zu vertreten.

Rechts. Von der Teilungsstelle des N. tibialis und N. peronaeus communis zweigt sich der N. cut. surae lat. ab, der einen mässig verästelten $Z$ weig nach lateral unten abgebend, mit dem schwachen $N$. cut. surae med. im mittleren Drittel des Unterschenkels anastomosiert, um den N. suralis zu vertreten.

Vergleich. Über das Auftreten der Anastomose sowie das Dickenverhältnis der beiden Wadennerven sind die vier Beine konkordant, und über die Höhe der Anastomose nur drei Beine, d.h. ausgenommen von dem rechten des II. Paarlings.

Fall 35, ZZ, s Monate.

I. Fetus, Nr. I 349, 今.

Beiderseits. Die beiden Wadennerven anastomosieren miteinander im mittleren Drittel des Unterschenkels, wobei der N. cut. surae lat. dicker als der N. cut. surae med. ist.

II. Fetus, Nr. 1350, 우.

Beiderseits. Die beiden Wadennerven anastomosieren miteinander im 
mittleren Drittel des Unterschenkels, wobei der N. cut. surae lat. dicker als der N. cut. surae med. ist.

Vergleich. Über die Nervenverteilung findet man eine ausgeprägte Konkordanz unter allen vier Beinen, an denen der stärkere N. cut. surae lat. mit dem schwächeren N. cut. surae med. im mittleren Drittel des L'nterschenkels anastomosiert.

Fall 36, ZZ, 4 Monate.

I. Fetus, Nr. 1426, 今.

Beiderseits. Die beiden Wadennerven anastomosieren miteinander im mittleren Drittel des Unterschenkels, wobei der N. cut. surae lat., der den kurzen vorderen Ast nach lateral unten absendet, dicker als der N. cut. surae med. ist.

II. Fetus, Nr. 1427, 우.

Beiderseits. Die beiden Wadennerven anastomosieren miteinander im unteren Drittel des Unterschenkels, wobei der N. cut. surae lat. dicker als der $\therefore$. cut. surae med. ist.

Vergleich. Über das Auftreten der Anastomose und das Dickenverhältnis der beiden Wadennerven zeigen alle vier Beine eine Konkordanz, obwohl dabei die Höhe der Anastomose bei beiden Paarlingen verschieden ist.

Fall 37, ZZ, 7 Monate.

I. Fetus, Nr. I450, 우.

Links. Die beiden Wadennerven anastomosieren miteinander im unteren Drittel des Unterschenkels, wobei der N. cut. surae lat. dicker als der N. cut. surae med. ist.

Rechts. Die beiden Wadennerven anastomosieren miteinander im unteren Drittel des Unterschenkels, wobei der N. cut. surae med. dicker als der N. cut. surae lat. ist.

II. Fetus, Nr. I451, 우.

Links. Die beiden Wadennerven anastomosieren miteinander hinter dem äusseren Knöchel, wobei der N. cut. surae med. dicker als der N. cut. surae lat. ist.

Rechts. Die beiden Wadennerven anastomosieren miteinander im mittleren Drittel des Unterschenkels, wobei der N. cut. surae med. dicker als der N. cut. surae lat. ist.

Vergleich. Über das Auftreten der Anastomose der beiden Wadennerven sind die vier Beine konkordant, obwohl deren Höhe nur an den beiden Beinen des I. Paarlings symmetrisch gleich ist. Über das Dickenverhältnis weisen drei Beine, d.h. ausgenommen von dem linken des I. Fetus, eine Konkordanz auf.

Fall $38, \mathrm{ZZ}, 7$ Monate. 
I. Fetus, Nr. 1463 , 今.

Links. Die beiden Wadennerven anastomosieren miteinander hinter dem äusseren Knöchel, wobei der N. cut. surae lat. dicker als der N. cut. surae med. ist.

Rechts. Die beiden Wadennerven anastomosieren miteinander im unteren Drittel des Unterschenkels, wobei der N. cut. surae lat., der einen kurzen Ast von seiner Wurzel zum oberen Wadenteil absendet, dicker als der N. cut. surae med. ist.

II. Fetus, Nr. 1464, 今.

Beiderseits. Der stärkere N. cut. surae med. vertritt den N. suralis. Der N. cut. surae lat. ist schwach; er gelangt, einige Äste nach beiderseits abgebend, auf das mittlere Drittel des Unterschenkels, ohne mit dem N. cut. surae med. $z u$ anastomosieren.

Vergleich. Über das Auftreten der Anastomose und das Dickenverhältnis der beiden Wadennerven bemerkt man eine Diskordanz zwischen den beiden Paarlingen. Die Höhe der Anastomose an den beiden Beinen beim I. Paarling ist auch ungleich.

Fall 39, ZZ, 7 Monate.

I. Fetus, Nr. 1465, 우.

Links. Die beiden gleich dicken Wadennerven anastomosieren miteinander hinter dem äusseren Knöchel.

Rechts. Der N. cut. surae lat. entspringt von zwei Wurzel aus dem N. peronaeus communis. Die beiden Wadennerven anastomosieren miteinander im unteren Drittel des Unterschenkels, wobei der N. cut. surae lat. dicker als der N. cut. surae med. ist.

II. Fetus, Nr. 1466, 우.

Links. Die beiden gleich dicken Wadennerven anastomosieren miteinander im unteren Drittel des Unterschenkels. Zwischen den beiden Wadennerven findet man einen feinen Ast aus dem $N$. cut. surae lat.

Rechts. Der schwächere N. cut. surae med. anastomosiert verdoppelt mit dem N. cut. surae lat. im unteren Drittel des Unterschenkels und hinter dem äusseren Knöchel. Zwischen den beiden Wadennerven findet man einen feinen Ast aus dem N. cut. surae lat.

Vergleich. Über das Auftreten der Anastomose der beiden Wadennerven findet man eine Konkordanz unter den vier Beinen, und über das Dickenverhältnis eine jederseitige Konkordanz zwischen den beiden Paarlingen. Über die Höhe der Anastomose weisen die vier Beine eine spiegelbildliche Konkordanz auf, ausgenommen von einer Doppelverbindung an dem rechten Beine des II. Paarlings.

Fall 40, ZZ, Io Monate. 
I. Fetus, Nr. 147r, 우.

Beiderseits. Die beiden Wadennerven anastomosieren miteinander im mittleren Drittel des Unterschenkels, wobei der N. cut. surae lat., der einen feinen Ast von dem Stamm entsendet, dicker als der N. cut. surae med. ist.

II. Fetus, Nr. 1472, 우.

Links. Die beiden Wadennerven anastomosieren miteinander im mittleren Drittel des Unterschenkels, wobei der N. cut. surae lat, der einen kurzen Ast von dem Stamm entsendet, dicker als der N. cut. surae med. ist.

Rechts. Die beiden Wadennerven anastomosieren miteinander im mittleren Drittel des Unterschenkels, wobei der N. cut. surae med. dicker als der $N$. cut. surae lat. ist, der einen kurzen Ast von dem Stamm entsendet.

Vergleich. Über das Auftreten und die Höhe der Anastomose der beiden Wadennerven sind die vier Beine konkordant. Über das Dickenverhältnis zeigen drei Beine, d.h. ausgenommen von dem rechten des II. Paarlings, eine Konkordanz.

Fall 4I, ZZ, 6 Monate.

I. Fetus, Nr. 1473, 우.

Beiderseits. Der N. cut. surae med. ist stärker ausgebildet und vertritt den $N$. suralis, wobei das Versorgungsgebiet des $N$. cut. surae lat. auf einen schmalen Hautteil auf dem lateralen Kopf des Wadenmuskels beschränkt ist.

II. Fetus, Nr. 1474, 우.

Links. Die beiden gleich dicken Wadennerven anastomosieren miteinander im mittleren Drittel des Unterschenkels. Der N. cut. surae lat. entsendet den feinen vorderen Ast von dem Stamm nach lateral unten.

Rechts. Die beiden Wadennerven anastomosieren miteinander im mittleren Drittel des Unterschenkels, wobei der N. cut. surae lat., der den feinen vorderen Ast von dem Stamm nach lateral unten absendet, dicker als der N. cut. surae med. ist.

Vergleich. Bei diesem Paar besteht eine ausgesprochene Diskordanz der Nervenverteilung, wenngleich sie bei jeden Paarlingen symmetrisch ganz ähnlich ist.

Fall 42, Eiigkeit unklar, 7 Monate.

I. Fetus, Nr. 540, 今.

Links. Die beiden gleich dicken Wadennerven anastomosieren miteinander im mittleren Drittel des Unterschenkels, um den N. suralis auszubilden.

Rechts. Die beiden Wadennerven anastomosieren miteinander im unteren Drittel des Unterschenkels, wobei der N. cut. surae lat., der den feinen vorderen Ast auf die obere Hälfte des Unterschenkels liefert, dicker als der $\mathrm{N}$. cut. surae med. ist.

II. Fetus, Nr. $54 \mathrm{I}$, $\hat{\delta}$. 
Links. Die beiden Wadennerven anastomosieren miteinander hinter dem äusseren Knöchel, wobei der N. cut. surae med. dicker als der N. cut. surae lat. ist.

Rechts. Die beiden gleich dicken Wadennerven anastomosieren miteinander im mittleren Drittel des Unterschenkels, um den N. suralis zu vertreten.

Vergleich. Über das Auftreten der Anastomose der beiden Wadennerven sind die vier Beine konkordant. Über das Dickenverhältnis und die Höhe der Anastomose findet man eine einseitige spiegelbildliche Konkordanz zwischen den beiden Paarlingen.

Fall 43, Eiigkeit unklar, 7 Monate.

I. Fetus, Nr. 571, 各.

Beiderseits. Die beiden Wadennerven anastomosieren miteinander im mittleren Drittel des Unterschenkels, wobei der N. cut. surae lat., der einige feine Ästchen absendet, dicker als der N. cut. surae med. ist.

II. Fetus, Nr. 572 , 今.

Beiderseits. Die beiden Wadennerven anastomosieren miteinander im mittleren Drittel des Unterschenkels, wobei der N. cut. surae lat., der einige feine Ästchen absendet, dicker als der N. cut. surae med. ist.

Vergleich. Die vier Beine weisen eine ausgeprägte Konkordanz untereinander auf, indem der stärkere $N$. cut. surae lat. mit dem N. cut. surae med. im mittleren Drittel des Unterschenkels sich vereinigt.

Fall 44, Eiigkeit unklar, 7 Monate.

I. Fetus, Nr. I003, 今.

Links. Die beiden Wadennerven anastomosieren miteinander im mittleren Drittel des Unterschenkels, wobei der N. cut. surae med. dicker als der N. cut. surae lat. ist.

Rechts. Die beiden Wadennerven anastomosieren miteinander im unteren Drittel des Unterschenkels, wobei der N. cut. surae lat., der den feinen vorderen Ast nach lateral unten absendet, dicker als der N. cut. surae med. ist.

II. Fetus, Nr. I004, ô.

Links. Die beiden Wadennerven anastomosieren miteinander im mittleren Drittel des Unterschenkels, wobei der N. cut. surae lat., der den feinen vorderen Ast von seinem Stamm nach lateral unten absendet, dicker als der N. cut. surae med. ist.

Rechts. Die beiden Wadennerven anastomosieren miteinander im unteren Drittel des Unterschenkels, wobei der N. cut. surae lat., der den feinen vorderen Ast von seinem Stamm nach lateral unten absendet, dicker als der N. cut. surae med. ist.

Vergleich. Die vier Beine sind konkordant hinsichtlich des Auftretens der Anastomose der beiden Wadennerven. Über die Höhe der Anastomose 
bemerkt man eine jederseitige Konkordanz und über das Dickenverhältnis eine Konkordanz unter drei Beinen, d.h. ausgenommen von dem linken des I. Paarlings.

Fall 45, Eiigkeit unklar, ro Monate.

I. Fetus, Nr. iros (I), ô.

Beiderseits. Die beiden Wadennerven anastomosieren miteinander im mittleren Drittel des Unterschenkels, wobei der N. cut. surae lat., der den feinen vorderen Ast von seinem Stamm nach lateral unten absendet, dicker als der $\mathrm{N}$. cut. surae med. ist.

II. Fetus, Nr. rios (II), 今.

Beiderseits. Die beiden Wadennerven anastomosieren miteinander im unteren Drittel des Unterschenkels, wobei der N. cut. surae med. dicker als der $\mathrm{N}$. cut. surae lat. ist, der den feinen vorderen Ast von seinem Stamm nach lateral unten absendet.

Vergleich. Über das Auftreten der Anastomose der beiden Wadennerven sind die vier Beine konkordant. Über die Höhe der Anastomose und das Dickenverhältnis findet man eine ausgeprägte Diskordanz zwischen den beiden Paarlingen.

\section{Zusammenfassung.}

Die Befunde hinsichtlich der drei Vergleichungsmerkmale, d.h. des Auftretens sowie der Höhe der Anastomose und der Dicke der beiden Wadennerven ( $N$. cutaneus surae lateralis und $N$. cutaneus surae medialis) sind in der Tabelle 2 zusammengestellt. Ich werde im folgenden, hauptsächlich die von mir umgerechneten Zahlen aus den Befunden von Ssokolow gegenüberstellend, die Ergebnisse mehr ausführlich darlegen.

I. Die Häufigkeit der Anastomose zwischen den beiden Wadennerven.

Die Häufigkeit der Anastomose zwischen den beiden Wadennerven an den untersuchten $\mathrm{r} 80$ Beinen ist mit denjenigen von Ssokolow an 500 Beinen, zusammen in der Tabelle 3 aufgeführt. Daraus kann man ersehen, dass die Häufigkeit der Anastomose bei den Zwillingsfeten an der linken Seite $(88.9 \% \pm 3.3 \mathrm{I})$ etwas grösser als an der rechten $(77.8 \% \pm 4.36) \mathrm{zu}$ sein scheint, und dass sie als Ganzes $83.3 \% \pm 2.78$ der Fälle ausmacht. Diese Häufigkeit ist etwas grösser als $52.6 \%$ (Ssokolow), 40.2\% (Kosinski) und $5 \mathrm{I} \%$ (Catania). Die Häufigkeit der Anastomose nach dem Geschlecht sowie der Eiigkeit ist ferner in der Tabelle 4 und s näher angegeben, woraus hervorgeht, dass die Häufigkeit durch das Geschlecht oder die Eiigkeit nicht wesentlich beeinflusst zu werden scheint. Wenn man nun die Häufigkeit der Anastomose auf den beiden Beinen in einzelnen Feten nach dem 
Tabelle 2. Zusammenfassung der Befunde.

\begin{tabular}{|c|c|c|c|c|c|c|c|c|c|}
\hline \multirow[t]{2}{*}{$\begin{array}{c}\text { Eiig- } \\
\text { keit }\end{array}$} & \multirow[t]{2}{*}{ Fall } & \multirow[t]{2}{*}{ Fetus } & \multirow[t]{2}{*}{$\begin{array}{l}\text { Ges- } \\
\text { chlecht }\end{array}$} & \multicolumn{2}{|c|}{$\begin{array}{c}\text { Anastomose } \\
\text { der } \\
\text { beiden } \\
\text { Wadennerven } \\
\end{array}$} & \multicolumn{2}{|c|}{$\begin{array}{l}\text { Höhe der } \\
\text { Anastomose }\end{array}$} & \multicolumn{2}{|c|}{$\begin{array}{c}\begin{array}{c}\text { Vergleich der Dicke } \\
\text { der beiden } \\
\text { Wadennerven }\end{array} \\
\end{array}$} \\
\hline & & & & 1 & $\mathrm{r}$ & 1 & $\mathbf{r}$ & 1 & $\mathrm{r}$ \\
\hline \multirow{26}{*}{$\mathrm{EZ}$} & I & II & $\delta$ & \pm & - & md & & $\begin{array}{l}\mathrm{L} \\
\text { LI }\end{array}$ & $\begin{array}{l}M \\
M\end{array}$ \\
\hline & 2 & II & 0 & $\begin{array}{l}+ \\
+\end{array}$ & + & $\begin{array}{l}\mathrm{md} \\
\mathrm{md}\end{array}$ & $\begin{array}{l}\text { ud } \\
\text { ud }\end{array}$ & $\begin{array}{l}\mathrm{L} \\
\mathrm{L}\end{array}$ & $\begin{array}{l}\mathrm{L} \\
\mathrm{L}\end{array}$ \\
\hline & 3 & $\begin{array}{l}\text { I } \\
\text { II }\end{array}$ & 우 & $\begin{array}{l}+ \\
+\end{array}$ & - & $\begin{array}{c}\mathrm{uk} \\
\mathrm{md}\end{array}$ & & $\begin{array}{l}M \\
M\end{array}$ & $\begin{array}{l}\mathrm{M} \\
\mathrm{M}\end{array}$ \\
\hline & 4 & $\begin{array}{l}\text { I } \\
\text { II }\end{array}$ & ऽ & + & $\begin{array}{l}+ \\
+\end{array}$ & $\begin{array}{l}\text { md } \\
\text { md }\end{array}$ & $\begin{array}{l}\mathrm{md} \\
\mathrm{md}\end{array}$ & $\begin{array}{r}M \\
\mathrm{LM}\end{array}$ & $\begin{array}{r}\mathrm{LM} \\
\mathrm{M}\end{array}$ \\
\hline & 5 & $\begin{array}{l}\text { I } \\
\text { II }\end{array}$ & 今 & $\begin{array}{l}+ \\
+\end{array}$ & + & $\begin{array}{l}\text { ud } \\
\text { ud }\end{array}$ & $\begin{array}{l}\text { md } \\
\text { md }\end{array}$ & $\begin{array}{l}\mathrm{L} \\
\mathrm{L}\end{array}$ & $\begin{array}{l}\bar{L} \\
\mathrm{~L}\end{array}$ \\
\hline & 6 & $\begin{array}{l}\text { I } \\
\text { II }\end{array}$ & q & + & - & $\begin{array}{l}\mathrm{md} \\
\left.\mathrm{od}^{2}\right)\end{array}$ & & $\begin{array}{l}\mathrm{L} \\
\mathrm{L}\end{array}$ & $\begin{array}{l}\mathrm{M} \\
\mathrm{M}\end{array}$ \\
\hline & 7 & $\begin{array}{l}\text { I } \\
\text { II }\end{array}$ & 우 & $\begin{array}{l}+ \\
+\end{array}$ & $\begin{array}{l}+ \\
+\end{array}$ & $\begin{array}{l}\mathrm{md} \\
\mathrm{ud}\end{array}$ & $\begin{array}{c}\text { md } \\
\text { ud }\end{array}$ & $\begin{array}{l}\mathrm{M} \\
\mathrm{M}\end{array}$ & $\begin{array}{c}\mathrm{L} M \\
\mathrm{~L}\end{array}$ \\
\hline & 8 & $\begin{array}{l}\text { I } \\
\text { II }\end{array}$ & 우 & $\begin{array}{l}+ \\
+\end{array}$ & $\overline{+}$ & $\begin{array}{l}\text { ud } \\
\text { ud }\end{array}$ & ud & $\begin{array}{l}\mathrm{L} \\
\mathrm{L}\end{array}$ & $\begin{array}{l}\mathrm{MI} \\
\mathrm{L}\end{array}$ \\
\hline & 9 & $\begin{array}{l}\text { I } \\
\text { II }\end{array}$ & ㅇ & $\begin{array}{l}+ \\
+\end{array}$ & + & $\begin{array}{l}\text { ud } \\
\text { md }\end{array}$ & $\begin{array}{l}\text { md } \\
\text { md }\end{array}$ & $\begin{array}{l}\mathrm{L} \\
\mathrm{L}\end{array}$ & $\mathrm{L}$ \\
\hline & 10 & II $^{-}$ & \& & \pm & + & md & md & $\begin{array}{l}\mathrm{L}^{-} \\
\mathrm{I}\end{array}$ & $\begin{array}{l}\mathrm{M} \\
\mathrm{M}\end{array}$ \\
\hline & I I & II & 今 & $\begin{array}{l}+ \\
+\end{array}$ & + & $\begin{array}{l}\mathrm{md} \\
\text { ud }\end{array}$ & $\begin{array}{l}\text { md } \\
\text { od }^{2 y}\end{array}$ & $\begin{array}{l}\mathrm{L} \\
\mathrm{L}\end{array}$ & $\begin{array}{l}\mathrm{L} \\
\mathrm{L}\end{array}$ \\
\hline & I 2 & II & $\delta$ & $\overline{-}$ & $\overline{-}$ & & & $\begin{array}{l}M \\
M\end{array}$ & $\begin{array}{l}M \\
M\end{array}$ \\
\hline & 13 & $\begin{array}{l}\text { I } \\
\text { II }\end{array}$ & q & $\begin{array}{l}+ \\
+\end{array}$ & $\begin{array}{l}+ \\
+\end{array}$ & $\begin{array}{l}\text { od }^{\prime \prime} \\
\text { md }\end{array}$ & $\begin{array}{l}\text { md } \\
\text { md }\end{array}$ & $\begin{array}{l}\mathrm{L} \\
\mathrm{L}\end{array}$ & $\begin{array}{l}\text { LMI } \\
\text { LMI }\end{array}$ \\
\hline & I 4 & II & $\delta$ & + & + & $\begin{array}{l}\mathrm{md} \\
\mathrm{md}\end{array}$ & $\begin{array}{l}\text { md } \\
\text { md }\end{array}$ & $\begin{array}{l}\mathrm{L} \\
\mathrm{L}\end{array}$ & $\begin{array}{l}\mathrm{L} \\
\mathrm{L}\end{array}$ \\
\hline & is & $\begin{array}{l}\text { I } \\
\text { II }\end{array}$ & $\hat{\delta}$ & $\begin{array}{l}+ \\
+\end{array}$ & + & $\begin{array}{l}\mathrm{md} \\
\mathrm{hk}\end{array}$ & $\begin{array}{l}\text { md } \\
\text { od") }^{n}\end{array}$ & $\begin{array}{l}\mathrm{LM} \\
\mathrm{LM}\end{array}$ & $\begin{array}{l}\text { LM } \\
\text { LM }\end{array}$ \\
\hline & 16 & $\begin{array}{l}\text { I } \\
\text { II }\end{array}$ & 우 & $\begin{array}{l}+ \\
+\end{array}$ & + & $\begin{array}{l}\text { ud } \\
\mathrm{hk}\end{array}$ & $\begin{array}{l}\text { ud } \\
\text { hk }\end{array}$ & $\begin{array}{l}\text { LM } \\
\text { LM }\end{array}$ & $\begin{array}{l}\text { LM } \\
\text { LMI }\end{array}$ \\
\hline & 17 & II & $\delta$ & 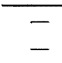 & $\overline{-}$ & & & $\begin{array}{l}M \\
M\end{array}$ & $\begin{array}{l}\mathrm{M} \\
\mathrm{M}\end{array}$ \\
\hline & 18 & $\begin{array}{l}\text { I } \\
\text { II }\end{array}$ & $\hat{\delta}$ & $\begin{array}{l}+ \\
+\end{array}$ & + & $\begin{array}{l}\mathrm{od}^{\prime \prime} \\
\mathrm{md}\end{array}$ & $\mathrm{md}$ & $\begin{array}{c}\mathrm{M} \\
\mathrm{L}\end{array}$ & $\begin{array}{l}\mathrm{L} \\
\mathrm{L}\end{array}$ \\
\hline & 19 & $\begin{array}{l}\text { I } \\
\text { II }\end{array}$ & $q$ & - & $\begin{array}{l}+ \\
+\end{array}$ & & $\begin{array}{l}\text { ud } \\
\text { ud }\end{array}$ & $\begin{array}{l}\mathrm{L} \\
\mathrm{L}\end{array}$ & $\begin{array}{l}\mathrm{L} \\
\mathrm{L}\end{array}$ \\
\hline & 20 & I & 今 & $\begin{array}{l}+ \\
+\end{array}$ & $\overline{-}$ & $\begin{array}{l}\text { md } \\
\text { md }\end{array}$ & & $\begin{array}{l}\mathrm{L} \\
\mathrm{L}\end{array}$ & $\begin{array}{l}M \\
\text { MI }\end{array}$ \\
\hline & $2 \mathrm{I}$ & $\begin{array}{l}\text { I } \\
\text { II }\end{array}$ & 우 & $\begin{array}{l}+ \\
+\end{array}$ & $\begin{array}{l}+ \\
+\end{array}$ & $\begin{array}{l}\text { uk } \\
\text { ud }\end{array}$ & $\begin{array}{l}\text { ud } \\
\text { ud }\end{array}$ & $\begin{array}{r}\mathrm{LM} \\
\mathrm{L}\end{array}$ & $\begin{array}{l}\mathrm{L} \\
\mathrm{L}\end{array}$ \\
\hline & 22 & I & $\hat{\delta}$ & $\begin{array}{l}+ \\
+\end{array}$ & + & $\begin{array}{l}\text { md } \\
\text { md }\end{array}$ & $\begin{array}{l}\text { ud } \\
\text { md }\end{array}$ & $\begin{array}{c}\mathrm{L} \\
\mathrm{LM}\end{array}$ & $\begin{array}{r}\mathrm{L} \\
\mathrm{L} . \mathrm{I}\end{array}$ \\
\hline & 23 & $\begin{array}{l}\text { II } \\
\text { II }\end{array}$ & $\delta$ & $\begin{array}{l}+ \\
+\end{array}$ & $\begin{array}{l}+ \\
+\end{array}$ & $\begin{array}{l}\text { ud } \\
\text { md }\end{array}$ & $\begin{array}{l}\text { ud } \\
\text { md }\end{array}$ & $\frac{L}{M}$ & $\begin{array}{r}\mathrm{L} \\
\mathrm{LN}\end{array}$ \\
\hline & 24 & II & $\widehat{\delta}$ & $\begin{array}{l}+ \\
+\end{array}$ & + & $\begin{array}{l}\mathrm{md} \\
\mathrm{md}\end{array}$ & $\begin{array}{l}\mathrm{md} \\
\mathrm{md}\end{array}$ & $\begin{array}{l}\mathrm{L} \\
\mathrm{L}\end{array}$ & $\begin{array}{l}\mathrm{L} \\
\mathrm{L}\end{array}$ \\
\hline & 25 & $\begin{array}{l}\text { I } \\
\text { II }\end{array}$ & $\hat{\delta}$ & $\begin{array}{l}+ \\
+\end{array}$ & $\begin{array}{l}+ \\
+\end{array}$ & $\begin{array}{l}\mathrm{md} \\
\mathrm{md}\end{array}$ & $\begin{array}{l}\mathrm{md} \\
\mathrm{md}\end{array}$ & $\begin{array}{l}\mathrm{L} \\
\mathrm{L}\end{array}$ & L. \\
\hline & 26 & $\begin{array}{l}\text { I } \\
\text { II }\end{array}$ & 今 & + & + & $\begin{array}{l}\mathrm{md}^{\prime \prime} \\
\text { od }^{\prime}\end{array}$ & $\begin{array}{l}\mathrm{md} \\
\left.\mathrm{od}^{2}\right)\end{array}$ & $\begin{array}{l}\mathrm{L} \\
\mathrm{L}\end{array}$ & $\begin{array}{l}\mathrm{L} \\
\mathrm{L}\end{array}$ \\
\hline
\end{tabular}


Tabelle 2.-(Fortsetzung)

\begin{tabular}{|c|c|c|c|c|c|c|c|c|c|}
\hline \multirow[t]{2}{*}{$\begin{array}{l}\text { Eiig- } \\
\text { keit }\end{array}$} & \multirow[t]{2}{*}{ Fall } & \multirow[t]{2}{*}{ Fetus } & \multirow[t]{2}{*}{$\begin{array}{l}\text { Ges- } \\
\text { chlecht }\end{array}$} & \multicolumn{2}{|c|}{$\begin{array}{c}\text { Anastomose } \\
\text { der } \\
\text { beiden } \\
\text { Wadennerven }\end{array}$} & \multicolumn{2}{|c|}{$\begin{array}{l}\text { Höhe der } \\
\text { Anastomose }\end{array}$} & \multicolumn{2}{|c|}{$\begin{array}{c}\text { Vergleich der Dicke } \\
\text { der beiden } \\
\text { Wadennerven }\end{array}$} \\
\hline & & & & 1 & $r$ & 1 & $r$ & 1 & $r$ \\
\hline \multirow[t]{8}{*}{$E Z$} & 27 & 1 & 잉 & $\begin{array}{l}+ \\
+\end{array}$ & $\begin{array}{l}+ \\
+\end{array}$ & $\begin{array}{c}\mathrm{ud}+\mathrm{uk} \\
\mathrm{ud}\end{array}$ & $\begin{array}{l}\mathrm{uk} \\
\mathrm{md}\end{array}$ & $\begin{array}{l}\mathrm{L} \\
\mathrm{L}\end{array}$ & $\begin{array}{l}\mathrm{L} \\
\mathrm{L}\end{array}$ \\
\hline & 28 & $\begin{array}{r}1 \\
\text { II }\end{array}$ & 市 & $\begin{array}{l}+ \\
+\end{array}$ & + & $\begin{array}{l}\text { md } \\
\text { md }\end{array}$ & $\begin{array}{l}\text { md } \\
\text { md }\end{array}$ & $\begin{array}{c}\mathrm{L} \\
\mathrm{LM}\end{array}$ & $\begin{array}{c}\mathrm{LM} \\
\mathrm{L}\end{array}$ \\
\hline & 29 & $\begin{array}{l}\text { I } \\
\text { II }\end{array}$ & $\begin{array}{l}\hat{0} \\
\text { \& }\end{array}$ & $\begin{array}{l}+ \\
+\end{array}$ & \pm & $\begin{array}{l}\text { md } \\
\text { ud }\end{array}$ & md & $\begin{array}{l}\mathrm{L} \\
\mathrm{L}\end{array}$ & $\begin{array}{l}\mathrm{L} \\
\mathrm{MI}\end{array}$ \\
\hline & 30 & I & $\begin{array}{l}\text { ㅇ } \\
\text { q }\end{array}$ & $\begin{array}{l}+ \\
+ \\
\end{array}$ & - & $\begin{array}{l}\text { ud } \\
\text { uk }\end{array}$ & & $\begin{array}{l}\mathrm{L} \\
\mathrm{L}\end{array}$ & $\begin{array}{l}\mathrm{L} \\
\mathrm{M}\end{array}$ \\
\hline & $3 I$ & II & $\begin{array}{l}\hat{D} \\
\hat{0}\end{array}$ & $\begin{array}{l}+ \\
+\end{array}$ & + & $\begin{array}{c}\text { md } \\
m d+u d\end{array}$ & $\begin{array}{l}\mathrm{hk} \\
\mathrm{ud}\end{array}$ & $\begin{array}{l}\mathrm{L} \\
\mathrm{L}\end{array}$ & $\mathrm{L}$ \\
\hline & 32 & II & $\begin{array}{l}\hat{\delta} \\
\text { q }\end{array}$ & $\begin{array}{l}+ \\
+\end{array}$ & + & $\begin{array}{l}\text { md } \\
\text { md }\end{array}$ & $\begin{array}{l}\text { md } \\
\text { md }\end{array}$ & $\begin{array}{l}\mathrm{L} \\
\mathrm{L}\end{array}$ & $\begin{array}{l}\mathrm{L} \\
\mathrm{L}\end{array}$ \\
\hline & 33 & II & $\begin{array}{l}\hat{\delta} \\
\hat{\delta}\end{array}$ & $\begin{array}{l}+ \\
+\end{array}$ & + & $\begin{array}{l}\text { ud } \\
\text { md }\end{array}$ & $\begin{array}{l}\text { ud } \\
\text { md }\end{array}$ & $\begin{array}{c}\mathrm{M} \\
\mathrm{L}\end{array}$ & $\begin{array}{l}\mathrm{L} \\
\mathrm{L}\end{array}$ \\
\hline & 34 & I & 克 & $\begin{array}{l}+ \\
+\end{array}$ & + & $\begin{array}{l}\text { ud } \\
\text { ud }\end{array}$ & $\begin{array}{l}\text { ud } \\
\text { md }\end{array}$ & $\begin{array}{l}\mathrm{L} \\
\mathrm{L}\end{array}$ & $\begin{array}{l}\mathrm{L} \\
\mathrm{L}\end{array}$ \\
\hline \multirow{9}{*}{$7 Z$} & 35 & $\begin{array}{r}\text { I } \\
\text { II }\end{array}$ & $\begin{array}{l}1 \\
+ \\
\end{array}$ & $\begin{array}{l}+ \\
+ \\
\end{array}$ & + & $\begin{array}{l}\text { md } \\
\text { md }\end{array}$ & $\begin{array}{l}\text { md } \\
\text { md }\end{array}$ & $\begin{array}{l}\mathrm{L} \\
\mathrm{L}\end{array}$ & $\begin{array}{l}\mathrm{L} \\
\mathrm{L}\end{array}$ \\
\hline & 36 & $\begin{array}{l}\mathrm{I} \\
\mathrm{HI}\end{array}$ & $\begin{array}{l}\delta \\
\text { के }\end{array}$ & $\begin{array}{l}+ \\
+\end{array}$ & $\begin{array}{l}+ \\
+\end{array}$ & $\begin{array}{l}\text { md } \\
\text { ud }\end{array}$ & $\begin{array}{l}\text { md } \\
\text { ud }\end{array}$ & $\begin{array}{l}\mathrm{L} \\
\mathrm{L}\end{array}$ & $\begin{array}{l}\mathrm{L} \\
\mathrm{L}\end{array}$ \\
\hline & 37 & $\begin{array}{r}\text { I } \\
\text { II }\end{array}$ & 우 & + & $\begin{array}{l}+ \\
+\end{array}$ & $\begin{array}{l}\text { ud } \\
\text { hk }\end{array}$ & $\begin{array}{l}\text { ud } \\
\text { md }\end{array}$ & $\mathrm{L}$ & $\begin{array}{l}\mathrm{MI} \\
\mathrm{M}\end{array}$ \\
\hline & 38 & $\begin{array}{l}\text { I } \\
\text { II }\end{array}$ & 今 & + & \pm & $\mathrm{hk}$ & ud & $\begin{array}{l}\mathrm{L} \\
\mathrm{M}\end{array}$ & $\begin{array}{l}\mathrm{L} \\
\mathrm{M}\end{array}$ \\
\hline & 39 & $\begin{array}{r}1 \\
\text { II }\end{array}$ & 웅 & $\begin{array}{l}+ \\
+\end{array}$ & $\begin{array}{l}+ \\
+\end{array}$ & $\begin{array}{l}\mathrm{hk} \\
\mathrm{ud}\end{array}$ & $\begin{array}{c}\mathrm{ud} \\
\mathrm{ud}+\mathrm{hk}\end{array}$ & $\begin{array}{l}\text { LM } \\
\text { LM }\end{array}$ & $\begin{array}{l}\mathrm{L} \\
\mathrm{L}\end{array}$ \\
\hline & 40 & $\begin{array}{l}\text { I } \\
\text { II }\end{array}$ & $\begin{array}{l}\text { 우 } \\
\text { 우 }\end{array}$ & + & $\begin{array}{l}+ \\
+\end{array}$ & $\begin{array}{l}\text { md } \\
\text { md }\end{array}$ & $\begin{array}{l}\mathrm{md} \\
\mathrm{md}\end{array}$ & $\begin{array}{l}\mathrm{L} \\
\mathrm{L}\end{array}$ & $\begin{array}{l}\mathrm{L} \\
\mathrm{II}\end{array}$ \\
\hline & 41 & II & $\begin{array}{l}\text { 우 } \\
\text { 우 }\end{array}$ & $\overline{+}$ & $\overline{+}$ & md & $\mathrm{md}$ & $\begin{array}{r}\text { MI } \\
\text { LM }\end{array}$ & $\begin{array}{c}\mathrm{M} \\
\mathrm{L}\end{array}$ \\
\hline & 42 & II & $\delta$ & + & $\begin{array}{l}+ \\
+\end{array}$ & $\begin{array}{l}\mathrm{md} \\
\mathrm{hk}\end{array}$ & $\begin{array}{l}\text { ud } \\
\text { md }\end{array}$ & $\begin{array}{r}\mathrm{LM} \\
\mathrm{M}\end{array}$ & L.M \\
\hline & 43 & II & $\begin{array}{l}\hat{\delta} \\
\delta\end{array}$ & $\begin{array}{l}+ \\
+\end{array}$ & + & $\begin{array}{l}\text { md } \\
\text { md }\end{array}$ & $\begin{array}{l}\text { md } \\
\text { md }\end{array}$ & $\begin{array}{l}\mathrm{L} \\
\mathrm{L}\end{array}$ & $\begin{array}{l}\mathrm{L} \\
\mathrm{L}\end{array}$ \\
\hline \multirow{2}{*}{$?$} & 44 & $\begin{array}{l}\text { l } \\
\text { II }\end{array}$ & 今 & + & + & $\begin{array}{l}\text { md } \\
\text { md }\end{array}$ & $\begin{array}{l}\text { ud } \\
\text { ud }\end{array}$ & $\stackrel{\mathrm{MI}}{\mathrm{L}}$ & $\begin{array}{l}\mathrm{L} \\
\mathrm{L}\end{array}$ \\
\hline & 45 & II & 今 & $\begin{array}{l}+ \\
+ \\
\end{array}$ & $\begin{array}{l}+ \\
+\end{array}$ & $\begin{array}{r}\text { md } \\
\text { ud }\end{array}$ & $\begin{array}{r}\text { md } \\
\text { ud }\end{array}$ & $\mathrm{L}$ & $\mathrm{L}$ \\
\hline
\end{tabular}

$\mathrm{l}=$ links, $\mathrm{r}=$ rechts, od $^{\prime}$ ) = In der Kniekehle (das obere Drittel des Unterschenkels), $\mathrm{od}^{2)}=$ - Ausserhalb der Kiniekehle (das obere Drittel des Unterschenkels), md $=$ Das mittlere Drittel des Unterschenkels, ud = Das untere Drittel des Unterschenkels, $\mathrm{hk}=$ Hinter dem äusseren Knöchel, uk = Unter dem äusseren Knöchel,

$\mathrm{L}=$ Der $\mathrm{N}$. cutaneus surac lateralis ist dicker als der $\mathrm{N}$. cutaneus surae medialis, II - Der N. cutaneus surae medialis ist dicker als der N. cutaneus surac lateralis, $\mathrm{LN}$ I $=$ Beide Wadennerven sind gleich dick. 
Tabelle 3. Anastomose zwischen dem N. cutaneus surae lat. und $\mathrm{N}$. cutaneus surae med.

\begin{tabular}{|c|c|c|c|c|c|c|}
\hline & \multicolumn{3}{|c|}{ Mogi } & \multicolumn{3}{|c|}{ Ssokolow } \\
\hline & $\begin{array}{c}\text { Links } \\
(9 \circ \text { Beine })\end{array}$ & $\begin{array}{c}\text { Rechts } \\
\text { (90 Beine) }\end{array}$ & $\begin{array}{c}\text { Summe } \\
(180 \text { Beine })\end{array}$ & $\begin{array}{c}\text { Links } \\
(25 \circ \text { Beine })\end{array}$ & $\begin{array}{c}\text { Rechts } \\
(250 \text { Beine })\end{array}$ & $\begin{array}{c}\text { Summe } \\
\text { (soo Beine) }\end{array}$ \\
\hline $\begin{array}{l}\text { Anastomose } \\
(+)\end{array}$ & $\begin{array}{c}80 \\
(88.9 \% \\
\pm 3.3 \mathrm{I})\end{array}$ & $\begin{array}{c}70 \\
(77.8 \% \\
\pm 4.36)\end{array}$ & $\begin{array}{c}x 50 \\
(83.3 \% \\
\pm 2.78)\end{array}$ & $\begin{array}{c}120 \\
(48.0 \%)\end{array}$ & $\begin{array}{c}143 \\
(57.2 \%\end{array}$ & $\begin{array}{c}263 \\
(52.6 \%)\end{array}$ \\
\hline $\begin{array}{c}\text { Anastomose } \\
(-)\end{array}$ & $\begin{array}{c}10 \\
(11.1 \% \\
\pm 3.31)\end{array}$ & $\begin{array}{c}20 \\
(22.2 \% \\
\pm 4.36)\end{array}$ & $\begin{array}{c}30 \\
(16.7 \% \\
\pm 2.78)\end{array}$ & $\begin{array}{c}130 \\
(52.0 \%)\end{array}$ & $\begin{array}{c}107 \\
(42.8 \%)\end{array}$ & $\begin{array}{c}237 \\
(47.4 \%)\end{array}$ \\
\hline
\end{tabular}

Tabelle 4. Die Häufigkeit der Anastomose zwischen dem

N. cutaneus surae lat. und N. cutaneus surae med. nach dem Geschlecht.

\begin{tabular}{|c|c|c|c|c|c|c|c|c|c|c|c|c|}
\hline Ma- & \multicolumn{6}{|c|}{ Mogi } & \multicolumn{6}{|c|}{ Ssokolow } \\
\hline & \multicolumn{3}{|c|}{$\hat{\delta}$} & \multicolumn{3}{|c|}{ 우 } & \multicolumn{3}{|c|}{$\delta$} & \multicolumn{3}{|r|}{ 우 } \\
\hline $\begin{array}{l}\text { Ana- } \\
\text { stomose }\end{array}$ & $\begin{array}{l}\mathrm{L} \\
55\end{array}$ & $\begin{array}{l}\mathrm{R} \\
55\end{array}$ & $\begin{array}{l}\text { Summe } \\
\text { I ro Beine }\end{array}$ & $\begin{array}{l}\mathrm{L} \\
35\end{array}$ & $\begin{array}{l}\mathrm{R} \\
35\end{array}$ & $\begin{array}{l}\text { Summe } \\
\text { 7o Beine }\end{array}$ & $\begin{array}{c}\mathrm{L} \\
130\end{array}$ & $\begin{array}{c}\mathrm{R} \\
\mathrm{I} 30\end{array}$ & $\begin{array}{l}\text { Summe } \\
260 \text { Beine }\end{array}$ & $\begin{array}{c}\mathrm{L} \\
120\end{array}$ & $\begin{array}{c}\mathrm{R} \\
\mathrm{I} 20\end{array}$ & $\begin{array}{c}\text { Summe } \\
240 \text { Beine }\end{array}$ \\
\hline$(+)$ & 49 & 45 & $\begin{array}{c}94 \\
(85.5 \% \\
\pm 3.36)\end{array}$ & $3 I$ & 25 & $\begin{array}{c}56 \\
(80.0 \% \\
\pm 4.77)\end{array}$ & 63 & 72 & $\begin{array}{c}135 \\
(51.9 \%)\end{array}$ & 57 & 71 & $\begin{array}{c}(28 \\
53.3 \%)\end{array}$ \\
\hline$(-)$ & 6 & IO & $\begin{array}{c}16 \\
(14.5 \% \\
\pm 3.36)\end{array}$ & 4 & 10 & $\begin{array}{c}14 \\
(20.0 \% \\
\pm 4.77) \\
\end{array}$ & $6_{7}$ & 58 & $\begin{array}{c}125 \\
(48.1 \%)\end{array}$ & 63 & 49 & $\begin{array}{c}\text { I I } 2 \\
(46.7 \% 0)\end{array}$ \\
\hline
\end{tabular}

$\mathrm{L}=$ links, $\mathrm{R}=$ rechts.

Tabelle 5. Die Häufigkeit der Anastomose zwischen dem $\mathrm{N}$. cutaneus surae lat. und $\mathrm{N}$. cutaneus surae med. nach der Eiigkeit.

\begin{tabular}{|c|c|c|c|c|c|c|c|c|c|}
\hline \multirow{2}{*}{$\begin{array}{l}\text { Ma- } \\
\text { terial } \\
\text { Ana- } \\
\text { stomose }\end{array}$} & \multicolumn{3}{|c|}{ E Z } & \multicolumn{3}{|c|}{ Z Z } & \multicolumn{3}{|c|}{ Eiigkeit unklar } \\
\hline & $\begin{array}{l}\mathrm{L} \\
54\end{array}$ & $\begin{array}{l}\mathrm{R} \\
54\end{array}$ & $\begin{array}{l}\text { Summe } \\
\text { Io8 Beine }\end{array}$ & $\begin{array}{l}\mathrm{L} \\
28\end{array}$ & $\begin{array}{l}R \\
28\end{array}$ & $\begin{array}{c}\text { Summe } \\
56 \text { Beine }\end{array}$ & $\begin{array}{l}\mathrm{L} \\
8 \\
\end{array}$ & $\begin{array}{r}R \\
8\end{array}$ & $\begin{array}{l}\text { Summe } \\
\text { I } 6 \text { Beine }\end{array}$ \\
\hline$(+)$ & 46 & 39 & $\begin{array}{c}85 \\
(78.7 \% \\
\pm 3.94)\end{array}$ & 26 & 23 & $\begin{array}{c}49 \\
(87.5 \% \\
\pm 4.4 \mathrm{I})\end{array}$ & 8 & 8 & $\begin{array}{c}16 \\
(100.0 \%)\end{array}$ \\
\hline$(-)$ & 8 & 15 & $\begin{array}{c}23 \\
(21.3 \% \\
\pm 3.94)\end{array}$ & 2 & 5 & $\begin{array}{c}7 \\
(12.5 \% \\
\pm 4.4 \mathrm{I})\end{array}$ & $\circ$ & $\circ$ & $\cdots$ \\
\hline
\end{tabular}

$\mathrm{L}=$ links, $\mathrm{R}=$ rechts.

Geschlecht sowie der Eiigkeit berechnet, so ergibt sich die Tabelle 6 und 7 . In der Tabelle 6 ist bemerkenswert, dass die asymmetrische Verteilung hinsichtlich des Auftretens der Anastomose bei weiblichen Feten $(34.3 \% \pm 8.00)$ etwas häufiger als bei männlichen $(7.3 \% \pm 3.5 \mathrm{I})$ vorkommt, die bei $\mathrm{S}$ s o k ol o w keinen merkwürdigen Unterschied aufweist. Sie zeigt ferner keinen nennenswerten statistischen Unterschied nach der Eiigkeit, wie die Tabelle 7 
Tabelle 6. Vergleich über die Anastomose zwischen den beiden Wadennerven in den einzelnen Feten nach dem Geschlecht.

\begin{tabular}{|c|c|c|c|c|c|}
\hline & $u$ & & Symmetrisch & & Asymmetrisch \\
\hline Geschlech & & $\begin{array}{c}\text { Bds. } \\
\text { Anastomose } \\
(+)\end{array}$ & $\begin{array}{c}\text { Bds. } \\
\text { Anastomose } \\
(-)\end{array}$ & Summe & $\begin{array}{c}\text { Eins. } \\
\text { Anastomose } \\
(+)\end{array}$ \\
\hline & $\begin{array}{c}\delta \\
\text { ( } 55 \text { Feten) }\end{array}$ & 45 & 6 & $\begin{array}{c}5 \mathrm{I} \\
(92.7 \% \pm 3.5 \mathrm{I})\end{array}$ & $(7.3 \% 4$ \\
\hline $\log 1$ & (35 $\stackrel{\text { F }}{\text { Feten })}$ & 22 & I & $\left(65.7 \%^{23} \pm 8.00\right)$ & $\begin{array}{c}12 \\
(34.3 \% \pm 8.00)\end{array}$ \\
\hline Sootolow & (1 зо Fälle) & 54 & 49 & $\begin{array}{c}103 \\
(79.2 \%)\end{array}$ & $\begin{array}{c}27 \\
(20.8 \%)\end{array}$ \\
\hline Ssokolow & (1 20 $\stackrel{\stackrel{9}{\text { Fälle) }})}{ }$ & $5 \mathrm{I}$ & 43 & $\begin{array}{c}94 \\
(78.3 \%)\end{array}$ & $\begin{array}{c}26 \\
(21.7 \%)\end{array}$ \\
\hline
\end{tabular}

Bds. $=$ Beiderseitig, $\quad$ Eins. $=$ Einseitig .

Tabelle 7. Vergleich über die Anastomose zwischen den beiden Wadennerven in den einzelnen Individuen nach der Eiigkeit.

\begin{tabular}{|c|c|c|c|c|}
\hline & \multicolumn{3}{|c|}{ Symmetrisch } & Asymmetrisch \\
\hline Eiigkeit & $\begin{array}{c}\text { Bds. } \\
\text { Anastomose } \\
(+)\end{array}$ & $\begin{array}{c}\text { Bds. } \\
\text { Anastomose } \\
(-)\end{array}$ & Summe & $\begin{array}{c}\text { Eins. } \\
\text { Anastomose } \\
(+)\end{array}$ \\
\hline $\begin{array}{c}\mathrm{EZ} \\
\left(s_{4} \text { Feten) }\right.\end{array}$ & 36 & 5 & $\begin{array}{c}4 \mathrm{I} \\
(75.9 \% \pm 5.8 \mathrm{I})\end{array}$ & $(24.1 \% \pm 5.81)$ \\
\hline $\begin{array}{c}\mathrm{ZZ} \\
\text { (28 Feten) }\end{array}$ & 23 & 2 & $\stackrel{25}{(89.3 \% \pm 5.84)}$ & $\left(10.7 \% \%^{3} \pm 5.84\right.$ \\
\hline
\end{tabular}

Bds. =-Beiderseitig, Eins. = Einseitig.

Tabelle 8. Vergleich über die Anastomose zwischen den beiden Wadennerven beim einzelnen $Z$ willingspaar.

\begin{tabular}{|c|c|c|c|c|}
\hline \multicolumn{2}{|c|}{ Eiigkeit } & $\begin{array}{c}\text { EZ } \\
\text { (27 Paare) }\end{array}$ & $\mid \begin{array}{c}Z Z \\
\text { (14 Paare) }\end{array}$ & $\begin{array}{l}\text { Unklar } \\
\text { (4 Paare) }\end{array}$ \\
\hline Anastomose $(+)$ an vier Beinen & Konkordanz & 17 & 10 & 4 \\
\hline Anastomose $(+)$ an drei Beinen & Diskordanz & 2 & I & $\rightarrow$ \\
\hline $\begin{array}{c}\text { Anastomose }(+) \text { an zwei Beinen } \\
\text { derselben Körperseite }\end{array}$ & Konkordanz & 4 & $\mathbf{I}$ & - \\
\hline $\begin{array}{c}\text { Anastomose }(+) \text { an zwei Beinen } \\
\text { eines Paarlings }\end{array}$ & Diskordanz & - & 2 & 一 \\
\hline $\begin{array}{l}\text { Anistomose }(+) \text { an zwei Beinen } \\
\text { in spiegelbildlicher Weise }\end{array}$ & $\begin{array}{c}\text { Spiegelbildliche } \\
\text { Konkordanz }\end{array}$ & $\mathbf{I}$ & 一 & - \\
\hline Anastomose $(+)$ an einem Bein & Diskordanz & I & - & - \\
\hline Ohne Arastomose & Konkordanz & 2 & - & - \\
\hline
\end{tabular}


lehrt. Verteilt man die untersuchten 45 Zwillingspaare in bezug auf dem Ähnlichkeitsgrad unter vier Beinen eines Paares, so unterrichtet hierüber die Tabelle 8. Bei der ungenügenden Zahl des Materials bin ich nicht in der Lage, einen zuverlässigen Unterschied zwischen den eineiigen und den zweieiigen Zwillingen und daraus eine erbbedingte Beziehung aufzustellen. Doch liegt es nach der Tabelle 8 näher, anzunehmen, dass die beiden Wadennerven im grössten Teil der Fälle (17/27 bei EZ, 10/14 bei ZZ) an allen vier Beinen eines Paares unabhängig von der Eiigkeit miteinander anastomosieren können.

Über die Verteilung der sonstigen Kombinationen sei hier auf die Beschreibung verzichtet.

2. Die Höhe der Anastomose zwischen den beiden Wadennerven.

Die Höhe der Anastomose zwischen den beiden Wadennerven an I 50 Beinen, auf denen die Anastomose stattfindet, ist in der Tabelle 9 näher aufgeführt. Daraus ist ersichtlich, dass die Zwillingsfeten sich nicht anders als die Einlinge (Erwachsene) zu verhalten scheinen, insofern, als die Vereinigungsstelle der beiden Wadennerven am häufigsten im mittleren Drittel des Unterschenkels vorkommt und dann die im unteren Drittel folgt. Es fällt aber auf, dass die Häufigkeit der Anastomose innerhalb der Kniekehle bei S sok olow bedeutend gross ( $1 \mathrm{r} .0 \%$ ) im Gegensatz zu meinen Fällen ( $1.3 \%$ ) ist. Kosinski (1926) hat die Vereinigungsstelle folgendermassen angegeben:

Tabelle 9. Höhe der Anastomose der beiden Wadennerven.

\begin{tabular}{|c|c|c|c|c|c|c|c|}
\hline \multicolumn{2}{|c|}{ Verfasser } & \multicolumn{3}{|c|}{ Mogi } & \multicolumn{3}{|c|}{ Ssokolow } \\
\hline \multicolumn{2}{|c|}{$\begin{array}{l}\text { Höhe der } \\
\text { Anastomose }\end{array}$} & $\begin{array}{c}\text { Links } \\
\text { (8o Beine) }\end{array}$ & $\begin{array}{c}\text { Rechts } \\
\text { (70 Beine) }\end{array}$ & $\begin{array}{c}\text { Summe } \\
\text { (I } 5 \circ \text { Beine) }\end{array}$ & $\begin{array}{c}\text { L. } \\
(\mathbf{I} 20)\end{array}$ & $\underset{(\mathrm{I} 43)}{\mathrm{R}}$ & $\begin{array}{c}\text { Summe } \\
\left(26_{3} \text { Beine }\right)\end{array}$ \\
\hline \multirow{2}{*}{$\begin{array}{c}\text { Das obere } \\
\text { Drittel des } \\
\text { Unterschenkels }\end{array}$} & $\begin{array}{l}\text { In der } \\
\text { Kniekehle }\end{array}$ & $\begin{array}{l}2 \\
(2.5 \% \\
\pm 1.75)\end{array}$ & $\cdots$ & $\begin{array}{c}2 \\
(1.3 \% \\
\pm 0.92) \\
\end{array}$ & 12 & 17 & $\begin{array}{c}29 \\
(\mathrm{I} 1.0 \%)\end{array}$ \\
\hline & $\begin{array}{c}\text { Ausserhalb } \\
\text { der Kniekehle }\end{array}$ & $\begin{array}{l}2 \\
(2.5 \% \\
\pm 1.75) \\
\end{array}$ & $\begin{array}{c}3 \\
(4.3 \% \\
\pm 2.42) \\
\end{array}$ & $\begin{array}{l}5 \\
(3.3 \% \\
\pm 1.46) \\
\end{array}$ & 2 & 5 & $(2.7 \%)$ \\
\hline \multicolumn{2}{|c|}{$\begin{array}{c}\text { Das mittlere Drittel des } \\
\text { Unterschenkels }\end{array}$} & $\begin{array}{r}45 \\
(56.3 \% \\
\pm 5.53) \\
\end{array}$ & $\begin{array}{r}4 \mathrm{I} \\
(58.6 \% \\
\pm 5.88) \\
\end{array}$ & $\begin{array}{r}86 \\
(57.4 \% \\
\pm 4.03) \\
\end{array}$ & 49 & 59 & $\begin{array}{c}108 \\
41.1 \%\end{array}$ \\
\hline \multicolumn{2}{|c|}{$\begin{array}{l}\text { Das untere Drittel des } \\
\text { Unterschenkels }\end{array}$} & $\begin{array}{c}20 \\
(25.0 \% \\
\pm 4.84) \\
\end{array}$ & $\begin{array}{c}22 \\
(31.4 \% \\
\pm 5.54) \\
\end{array}$ & $\begin{array}{r}42 \\
(28.0 \% \\
\pm 3.66) \\
\end{array}$ & 48 & 52 & $\begin{array}{c}100 \\
(38.0 \%) \\
\end{array}$ \\
\hline \multicolumn{2}{|c|}{ Hinter dem äusseren Knöchel } & $\begin{array}{c}6 \\
(7.5 \% \\
\pm 2.95) \\
\end{array}$ & $\begin{array}{c}2 \\
2.9 \% \\
\pm 2.01) \\
\end{array}$ & $\begin{array}{c}8 \\
(5.3 \% \\
\pm 1.83) \\
\end{array}$ & 2 & 2 & $\left(\begin{array}{c}4 \\
(1.5 \%\end{array}\right.$ \\
\hline \multicolumn{2}{|c|}{ Unter dem äusseren Knöchel } & $\begin{array}{l}3 \\
(3.7 \% \\
\pm 2 . \mathrm{II}) \\
\end{array}$ & $\begin{array}{c}\mathrm{I} \\
(\mathrm{I} .4 \% \\
\pm \mathrm{I} .4 \mathrm{I}) \\
\end{array}$ & $\begin{array}{c}4 \\
(2.7 \% \\
\pm 1.32) \\
\end{array}$ & 4 & 4 & $\begin{array}{c}8 \\
(3.0 \%)\end{array}$ \\
\hline \multicolumn{2}{|c|}{ Doppelanastomose } & $\begin{array}{l}2 \\
(2.5 \% \\
\pm 1.75)\end{array}$ & $\begin{array}{c}1 \\
(1.4 \% \\
\pm 1.4 \mathrm{I}) \\
\end{array}$ & $\begin{array}{l}3 \\
(2.0 \% \\
\pm 1.14) \\
\end{array}$ & 3 & 4 & $(2.7 \%)$ \\
\hline
\end{tabular}


$1.3 \%$ am obersten Viertel, $18.6 \%$ am zweiten Viertel, $65.1 \%$ am dritten Tiertel und $7.0 \%$ am untersten Viertel des Unterschenkels. Diese Ergebnisse timmen also ohne Zweifel mit den unseren gut überein, indem sie lehren, lass die Vereinigung der beiden Wadennerven am häufigsten an dem mittleren tbschnitt des Unterschenkels stattfindet. Die Höhe der Anastomose ist erner, nach dem Geschlecht sowie der Eiigkeit eingeteilt, in der Tabelle ro Ind I I näher gezeigt. Daraus kann man ersehen, dass die Höhe bei weiblichen

Tabelle iо. Höhe der Anastomose der beiden Wadennerven nach dem Geschlecht.

\begin{tabular}{|c|c|c|c|c|c|c|c|}
\hline \multirow{2}{*}{\multicolumn{2}{|c|}{$\begin{array}{l}\begin{array}{c}\text { Höhe der } \\
\text { Anastomose }\end{array} \\
\text { Material }\end{array}$}} & \multicolumn{3}{|c|}{ 今 } & \multicolumn{3}{|c|}{ 우 } \\
\hline & & $\begin{array}{l}\mathrm{L} \\
49\end{array}$ & $\begin{array}{l}\mathrm{R} \\
45 \\
\end{array}$ & $\begin{array}{l}\text { Summe } \\
94 \text { Beine }\end{array}$ & $\begin{array}{l}\mathrm{L} \\
3 \mathrm{I}\end{array}$ & $\begin{array}{l}\mathrm{R} \\
25\end{array}$ & $\begin{array}{l}\text { Summe } \\
56 \text { Beine }\end{array}$ \\
\hline \multirow{2}{*}{$\begin{array}{l}\text { Das obere } \\
\text { Drittel des } \\
\text { Unterschenkels }\end{array}$} & $\begin{array}{c}\text { In der } \\
\text { Kniekehle }\end{array}$ & $\mathbf{I}$ & 0 & $\begin{array}{c}I \\
(1.1 \% \\
\pm 1.07)\end{array}$ & I & ० & $\begin{array}{l}I \\
(1.8 \% \\
\pm 1.78)\end{array}$ \\
\hline & $\begin{array}{c}\text { Ausserhalb } \\
\text { der Kniekehle }\end{array}$ & I & 3 & $\begin{array}{c}4 \\
(4.2 \% \\
\pm 2.03)\end{array}$ & $\mathbf{I}$ & $\circ$ & $\begin{array}{c}\mathrm{I} \\
(\mathrm{I} .8 \% \\
\pm \mathrm{I} .78)\end{array}$ \\
\hline \multicolumn{2}{|c|}{$\begin{array}{l}\text { Das mittlere Drittel des } \\
\text { Unterschenkels }\end{array}$} & 33 & 28 & $\begin{array}{c}6 \mathrm{I} \\
(64.9 \% \\
\pm 4.92)\end{array}$ & I 2 & 13 & $\begin{array}{c}25 \\
(44.6 \% \\
\pm 6.63)\end{array}$ \\
\hline \multicolumn{2}{|c|}{$\begin{array}{l}\text { Das untere Drittel des } \\
\text { Unterschenkels }\end{array}$} & 9 & I 2 & $\begin{array}{c}21 \\
(22.3 \% \\
\pm 4.30) \\
\end{array}$ & I I & 10 & $\begin{array}{c}21 \\
(37.5 \% \\
\pm 6.46) \\
\end{array}$ \\
\hline \multicolumn{2}{|c|}{ Hinter dem äusseren Knöchel } & 3 & I & $\begin{array}{c}4 \\
(4.2 \% \\
\pm 2.03) \\
\end{array}$ & 3 & $\mathbf{I}$ & $\begin{array}{c}4 \\
(7.1 \% \\
\pm 3.43) \\
\end{array}$ \\
\hline \multicolumn{2}{|c|}{ Unter dem äusseren Knöchel } & ० & I & $\begin{array}{c}1 \\
(1.1 \% \\
\pm 1.07)\end{array}$ & 3 & ○ & $\begin{array}{c}3 \\
(5.4 \% \\
\pm 3.02)\end{array}$ \\
\hline \multicolumn{2}{|c|}{ Doppelanastomose } & 2 & o & $\begin{array}{c}2 \\
(2.2 \% \\
\pm 1.5 \mathrm{I})\end{array}$ & ○ & I & $\begin{array}{c}1 \\
(1.8 \% \\
\pm 1.78)\end{array}$ \\
\hline
\end{tabular}

$\mathrm{L}=$ links, $\quad \mathrm{R}=$ rechts.

zeten sich mehr distal als bei männlichen verschiebt, obwohl sie dabei nach der Iiigkeit keinen nennenswerten Unterschied aufweist. Wenn man nun die łäufigkeit der symmetrischen und asymmetrischen Verteilung der Höhe auf len beiden Beinen in den einzelnen Feten nach dem Geschlecht sowie der ziigkeit berechnet, so ergibt sich die Tabelle ${ }_{2}$ und I 3. Aus diesen Tabellen :rhellt, dass der Prozentsatz der Feten, bei denen die Höhe der Anastomose der seiden Wadennerven beiderseits gleich ist, etwa 2 mal häufiger als die der zeten, bei denen die Höhe beiderseits ungleich ist, was auch von dem Geschecht oder Eiigkeit unabhängig ist. Verteilt man die untersuchten 45 Zwilingspaaren in Bezug auf dem Ähnlichkeitsgrad unter den vier Beinen eines 'aares, so gibt die Tabelle 14 Aufschluss. Daraus ersieht man, dass die töhe keineswegs ein Vergleichungsmerkmal für die auffallende Konkordanz, 
Tabelle Ir. Höhe der Anastomose der beiden Wadennerven nach der Eiigkeit.

\begin{tabular}{|c|c|c|c|c|c|c|c|c|c|c|}
\hline \multirow{2}{*}{\multicolumn{2}{|c|}{$\begin{array}{l}\begin{array}{c}\text { Höhe der } \\
\text { Anastomose }\end{array} \\
\end{array}$}} & \multicolumn{3}{|c|}{$\mathrm{EZ}$} & \multicolumn{3}{|c|}{$\mathrm{ZZ}$} & \multicolumn{3}{|c|}{ Eiigkeit unklar } \\
\hline & & $\begin{array}{l}\mathrm{L} \\
46\end{array}$ & $\begin{array}{c}\mathrm{R} \\
39\end{array}$ & $\begin{array}{c}\text { Summe } \\
85 \text { Beine }\end{array}$ & $\begin{array}{l}\mathrm{L} \\
26\end{array}$ & $\begin{array}{l}\mathrm{R} \\
23 \\
\end{array}$ & $\begin{array}{l}\text { Summe } \\
49 \text { Beine }\end{array}$ & $\begin{array}{l}\mathrm{L} \\
8\end{array}$ & $\begin{array}{l}\mathrm{R} \\
8\end{array}$ & $\begin{array}{l}\text { Summe } \\
16 \text { Beine }\end{array}$ \\
\hline \multirow{2}{*}{\begin{tabular}{c|} 
Das obere \\
Drittel des \\
Unterschenkels
\end{tabular}} & $\begin{array}{l}\text { In der } \\
\text { Kniekehle }\end{array}$ & 2 & 0 & $\begin{array}{c}2 \\
\left(2.4^{2 \prime}\right. \\
\pm 1.66) \\
\end{array}$ & 0 & 0 & - & 0 & 0 & - \\
\hline & $\begin{array}{c}\text { Ausserhalb } \\
\text { der Kniekehle }\end{array}$ & 2 & 3 & $\begin{array}{l}5 \\
(5.9 \% \\
\pm 2.55)\end{array}$ & 0 & $\circ$ & $\cdots$ & 0 & 0 & - \\
\hline \multicolumn{2}{|c|}{$\begin{array}{c}\text { Das mittlere Drittel des } \\
\text { Unterschenkels }\end{array}$} & 26 & 23 & $\begin{array}{c}49 \\
(57.6 \% \\
\pm 5.35) \\
\end{array}$ & 13 & $\mathrm{I} 4$ & $\begin{array}{l}27 \\
55.1 \% \\
\pm 7.11 \\
\end{array}$ & 6 & 4 & $\begin{array}{c}10 \\
(62.5 \% \\
\pm 12.21) \\
\end{array}$ \\
\hline \multicolumn{2}{|c|}{$\begin{array}{l}\text { Das untere Drittel des } \\
\text { Unterschenkels }\end{array}$} & I I & I I & $\begin{array}{l}22 \\
\left(25.9^{\circ}\right. \\
\pm 4.25) \\
\end{array}$ & 8 & 7 & $\begin{array}{c}15 \\
(30.6 \% \\
\pm 6.57)\end{array}$ & I & 4 & $\begin{array}{c}5 \\
(31.3 \% \\
\pm 11.60) \\
\end{array}$ \\
\hline \multicolumn{2}{|c|}{$\begin{array}{l}\text { Hinter dem äusseren } \\
\text { Knöchel }\end{array}$} & 2 & I & $\begin{array}{l}3 \\
\left(3.5^{\circ} \circ\right. \\
\pm x .99)\end{array}$ & 3 & I & $\begin{array}{c}4 \\
(8.2 \% \\
\pm 3.92)\end{array}$ & I & $\circ$ & $\begin{array}{c}1 \\
(6.2 \% \\
\pm 6.03) \\
\end{array}$ \\
\hline \multicolumn{2}{|c|}{$\begin{array}{l}\text { Unter dem äusseren } \\
\text { Knöchel }\end{array}$} & 2 & I & $\begin{array}{l}3 \\
(3.5 \% \\
\pm 1.99)\end{array}$ & I & 0 & $\begin{array}{c}\mathrm{I} \\
2.0 \% \\
\pm 2.00)\end{array}$ & $\circ$ & 0 & - \\
\hline \multicolumn{2}{|c|}{ Doppelanastomose } & I & 0 & $\begin{array}{c}\mathrm{I} \\
(\mathrm{r} .2 \% \\
\pm \mathrm{I} .39) \\
\end{array}$ & $\mathbf{I}$ & I & $\begin{array}{c}2 \\
(4.1 \% \\
\pm 2.83\end{array}$ & $\circ$ & 0 & - \\
\hline
\end{tabular}

$\mathrm{L}=$ links, $\mathrm{R}=$ rechts.

Tabelle r2. Vergleich über die Höhe der Anastomose der beiden Wadennerven in den einzelnen Individuen nach dem Geschlecht.

\begin{tabular}{|c|c|c|}
\hline $\begin{array}{c}\text { Höhe der } \\
\text { Anastomose }\end{array}$ & $\begin{array}{c}\text { Beiderseits } \\
\text { gleich }\end{array}$ & $\begin{array}{l}\text { Beiderseits } \\
\text { ungleich }\end{array}$ \\
\hline (45 Feten) & $\begin{array}{c}28 \\
(62.2 \% \\
\pm 7.22)\end{array}$ & $\begin{array}{c}17 \\
(37.8 \% \\
\pm 7.22)\end{array}$ \\
\hline (22 $\stackrel{\text { F }}{\text { Feten })}$ & $\begin{array}{c}16 \\
\left(72.7^{\circ}\right)^{\prime} \\
\pm 9.48)\end{array}$ & $\begin{array}{c}6 \\
(27.3 \% \\
\pm 9.48)\end{array}$ \\
\hline
\end{tabular}

Tabelle I3. Vergleich über die Höhe der Anastomose der beiden Wadennerven in den einzelnen Individuen nach der Eiigkeit.

\begin{tabular}{|c|c|c|}
\hline Höhe der & $\begin{array}{l}\text { Beiderseits } \\
\text { gleich }\end{array}$ & $\begin{array}{l}\text { Beiderseits } \\
\text { ungleich }\end{array}$ \\
\hline $\begin{array}{c}\text { EZ } \\
\text { (36 Feten) }\end{array}$ & $\begin{array}{r}23 \\
(63.9 \% \\
\pm 7.681 \\
\end{array}$ & $\begin{array}{c}13 \\
(36.1 \% \\
\pm 7.68) \\
\end{array}$ \\
\hline$\left(\begin{array}{c}\mathrm{ZZ} \\
\text { (23 Feten) }\end{array}\right.$ & $\begin{array}{c}16 \\
(69.6 \% \\
\pm 9.59)\end{array}$ & $\begin{array}{c}7 \\
(30.4 \% \\
\pm 9.59)\end{array}$ \\
\hline
\end{tabular}

im Gegensatz zum ersten Merkmal (über das Auftreten der Anastomose) zu sein scheint, weil man erkennt, dass die Zahlen in der Tabelle 14 mehr oder weniger gleichmässig verteilt sind.

3. Die Dicke der beiden Wadennerven.

Die Ergebnisse über den Dickenvergleich der beiden Wadennerven an i 80 (Mogi), soo (Ssokolow) und so Beine (Catania) sind in der Tabelle is zusammengestellt. Es fällt zunächst in der Tabelle auf, dass die Häufigkeit 
Tabelle It. Vergleich über die Höhe der Anastomose der beiden Wadennerven beim einzelnen Zwillingspaar.

\begin{tabular}{|c|c|c|c|c|}
\hline Howhe der Anastomose & $\begin{array}{l}\text { venanastomose } \\
\text { ier Beinen }\end{array}$ & $\begin{array}{c}\mathrm{EZ} \\
\text { (17 Paare) }\end{array}$ & $\begin{array}{c}\mathrm{ZZ} \\
\text { (so Paare) }\end{array}$ & $\begin{array}{c}? \\
\text { (4 Paare) }\end{array}$ \\
\hline Gleich an vier Beinen & Konkordanz & 4 & 4 & I \\
\hline Gleich an drei Beinen & Diskordanz & 4 & $\mathbf{I}$ & -- \\
\hline $\begin{array}{c}\text { Gleich an zwei Beinen derselben } \\
\text { Körperseite }\end{array}$ & Konkordanz & 2 & $\cdots$ & I \\
\hline Spiegelbildlich gleich an zwei Beinen & $\begin{array}{l}\text { Spiegelbildliche } \\
\text { Konkordanz }\end{array}$ & $\ldots$ & $\ldots$ & -.- \\
\hline Gleich an zwei Beinen jedes Paarlings & Diskordanz & 4 & 2 & I \\
\hline Sonstige & Diskordanz & 3 & 3 & I \\
\hline
\end{tabular}

Tabelle is. Vergleich der Dicke der beiden Wadennerven.

\begin{tabular}{|c|c|c|c|c|c|c|c|c|}
\hline \multicolumn{2}{|c|}{ Verfasser } & \multicolumn{3}{|c|}{ Mogi } & \multicolumn{3}{|c|}{ Ssokolow } & \multirow{2}{*}{$\begin{array}{c}\text { Catania } \\
\text { Summe } \\
\text { so Beine }\end{array}$} \\
\hline \multirow{4}{*}{$\begin{array}{c}\text { Ana- } \\
\text { stomos: } \\
(+)\end{array}$} & & $\begin{array}{c}\text { Links } \\
(9 \circ \text { Beine) }\end{array}$ & $\begin{array}{c}\text { Rechts } \\
(9 \circ \text { Beine) }\end{array}$ & $\begin{array}{l}\text { Summe } \\
\text { (I } 80 \text { Beine }\end{array}$ & $\begin{array}{c}\text { Links } \\
250 \text { Beine }\end{array}$ & $\begin{array}{c}\text { Rechts } \\
\text { 25o Beine }\end{array}$ & $\begin{array}{c}\text { Summe } \\
\text { soo Beine }\end{array}$ & \\
\hline & Lat $>$ Med & $\begin{array}{r}56 \\
(62.3 \% \\
\pm 5.10) \\
\end{array}$ & $\begin{array}{r}52 \\
(57.8 \% \\
\pm 5.20\end{array}$ & $\begin{array}{c}108 \\
(60.0 \% \\
\pm 3.65) \\
\end{array}$ & $\begin{array}{c}44 \\
(17.6 \%)\end{array}$ & $(16.8 \%)$ & $\begin{array}{c}86 \\
\left(17.2^{\circ}\right)\end{array}$ & 6\%ó \\
\hline & Lat $=$ Med & $\begin{array}{c}12 \\
(13.3 \% \\
\pm 3.58)\end{array}$ & $\begin{array}{c}12 \\
(13.3 \% \\
\pm 3.58\end{array}$ & $\begin{array}{c}24 \\
(13.3 \% \\
\pm 2.53)\end{array}$ & $\begin{array}{c}5 \mathrm{I} \\
(20.4 \%)\end{array}$ & $\begin{array}{c}67 \\
(26.8 \%\end{array}$ & $\begin{array}{c}118 \\
(23.6 \% 0)\end{array}$ & $16 \%$ \\
\hline & Lat $<$ Med & $\begin{array}{c}12 \\
\left(13.3^{\circ}=\right. \\
\pm 3.58) \\
\end{array}$ & $\begin{array}{c}6 \\
(6.7 \% \\
\pm 2.64)\end{array}$ & $\begin{array}{c}18 \\
\left(10.0^{\circ} \%\right. \\
\pm 2.22) \\
\end{array}$ & $\begin{array}{c}25 \\
(10.0 \%)\end{array}$ & $\begin{array}{c}34 \\
(13.6 \% \%)\end{array}$ & $\begin{array}{c}59 \\
(11.8 \%\end{array}$ & $35^{\circ} \%$ \\
\hline \multirow{2}{*}{$\begin{array}{c}\text { Ana- } \\
\text { stomose } \\
(-)\end{array}$} & Lat $=$ Med & $\begin{array}{c}2 \\
\left(2.2^{\circ} o^{\prime}\right. \\
\pm 1.54\end{array}$ & $\begin{array}{c}2 \\
2.2 \% \\
\pm 1.54\end{array}$ & $\begin{array}{c}4 \\
(2.2 " \prime \\
\pm 1.09)\end{array}$ & $\begin{array}{c}\text { II } \\
\left(4.4^{\circ} 0\right) \\
\end{array}$ & $\left(\begin{array}{c}7 \\
(2.8 \%\end{array}\right)$ & $\begin{array}{c}18 \\
(3.6 \%)\end{array}$ & $14 \%$ \\
\hline & Lat $<$ Med & $\begin{array}{c}8 \\
(8.9 \% \\
\pm 2.02) \\
\end{array}$ & $\begin{array}{r}18 \\
20.0 \% \\
\pm 4.2 \mathrm{I} \\
\end{array}$ & $\begin{array}{c}26 \\
(14.5 \% \\
\pm 2.63) \\
\end{array}$ & $\begin{array}{c}119 \\
(47.6 \%)\end{array}$ & $\begin{array}{c}100 \\
(40.0 \%)\end{array}$ & $\begin{array}{c}219 \\
\left(43.8^{\circ}, 0\right)\end{array}$ & $29 \%$ \\
\hline
\end{tabular}

Lat $=\mathrm{N}$. cutaneus surae lateralis, $\mathrm{Med}=\mathrm{N}$. cutaneus surac medialis.

der Kombination von ,. Anastomose $(+)$, Lat $>$ Med “ bei den Zwillingsfeten $(60.0 \%)$ bedeutend grösser als diejenige von S sokolow $(17.2 \%)$ und Catania $(6 \%)$ ist, und dass die Kombination von "Anastomose (-), Lat $<$ Med " bei Ssokolow (43.8\%) und, Anastomose (+), Lat<Med" bei Catania $(35 \%)$ am häufigsten vorkommt. Die Dickenverhältnisse sind ferner, nach dem Geschlecht sowie der Eiigkeit eingeteilt, in der Tabelle 16 und 17 näher gezeigt. Auch in diesen Tabellen kann man die eben gesagten Tatsachen feststellen, indem man keinen nennenswerten Unterschied unter 
Tabelle 16. Vergleich der Dicke der beiden Wadennerven nach dem Geschlecht.

\begin{tabular}{|c|c|c|c|c|c|c|c|c|c|c|c|c|c|}
\hline \multirow{3}{*}{\multicolumn{2}{|c|}{ Merfasser }} & \multicolumn{6}{|c|}{ Mogi } & \multicolumn{6}{|c|}{ Ssokolow } \\
\hline & & \multicolumn{3}{|c|}{ \$ } & \multicolumn{3}{|c|}{ 우 } & \multicolumn{3}{|c|}{ ڤ } & \multicolumn{3}{|c|}{ 우 } \\
\hline & & $\begin{array}{l}\mathrm{L} \\
55\end{array}$ & $\begin{array}{l}\mathrm{R} \\
55\end{array}$ & $\begin{array}{c}\text { Summe } \\
\text { I IO }\end{array}$ & $\begin{array}{l}\mathrm{L} \\
35\end{array}$ & $\begin{array}{l}\mathrm{R} \\
35\end{array}$ & $\int_{70}^{\text {Summe }}$ & $\underset{I 30}{L}$ & $\underset{130}{R}$ & $\begin{array}{c}\text { Summe } \\
260\end{array}$ & $\begin{array}{c}\mathrm{L} \\
\mathrm{I} 20\end{array}$ & $\begin{array}{c}\mathrm{R} \\
\mathrm{I} 20\end{array}$ & $\underset{240}{\text { Summe }}$ \\
\hline \multirow{3}{*}{ 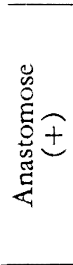 } & Lat $>$ Med & 36 & 37 & $\begin{array}{c}73 \\
(66.4 \% \\
\pm 4.50)\end{array}$ & 20 & Is & $\begin{array}{c}35 \\
(50.0 \% \\
\pm 5.96)\end{array}$ & 23 & 19 & $\begin{array}{c}42 \\
\text { (16.1\%) }\end{array}$ & $2 I$ & 23 & $\begin{array}{c}44 \\
(18.3 \%)\end{array}$ \\
\hline & Lat $=$ Med & 6 & 6 & $\begin{array}{c}12 \\
(10.9 \% \\
\pm 2.85) \\
\end{array}$ & 6 & 6 & $\begin{array}{c}12 \\
(17.1 \% \\
\pm 4.49) \\
\end{array}$ & 29 & 38 & $\begin{array}{c}67 \\
(25.8 \%)\end{array}$ & 22 & 29 & $\begin{array}{c}51 \\
\left(21.3^{\circ} 0^{\prime}\right.\end{array}$ \\
\hline & Lat $<$ Med & 7 & 2 & $\begin{array}{c}9 \\
(8.2 \% \\
\pm 2.62)\end{array}$ & 5 & 4 & $\begin{array}{c}9 \\
\text { (1 } 2.9 \% \\
\pm 4.0 \mathrm{I})\end{array}$ & II & Is & $\begin{array}{c}26 \\
(10.0 \%)\end{array}$ & I 4 & 19 & $\left(\begin{array}{c}33 \\
(13.7 \%)\end{array}\right.$ \\
\hline \multirow{2}{*}{ 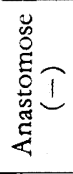 } & Lat $>$ Med & 0 & I & $\begin{array}{c}I \\
(0.9 \% \\
\pm 0.90)\end{array}$ & 2 & I & $\begin{array}{c}3 \\
(4.3 \% \\
\pm 2.42)\end{array}$ & 5 & 4 & $(3.5 \%)$ & 6 & 3 & $(3.8 \%)$ \\
\hline & Lat $<$ Med & 6 & 9 & $\begin{array}{c}15 \\
(13.6 \% \\
\pm 3.40)\end{array}$ & 2 & 9 & $\begin{array}{c}\text { I I } \\
(15.7 \% \\
\pm 4.34)\end{array}$ & 62 & 54 & $\begin{array}{c}116 \\
(44.6 \%)\end{array}$ & 57 & 46 & $\begin{array}{c}103 \\
(42.9 \%)\end{array}$ \\
\hline
\end{tabular}

$\mathrm{L}=$ links, $\mathrm{R}=$ rechts, $\mathrm{Lat}=\mathrm{N}$. cutaneus surae lateralis, $\mathrm{Med}=\mathrm{N}$. cutaneus surae medialis.

Tabelle i7. Vergleich der Dicke der beiden Wadennerven nach der Eiigkeit.

\begin{tabular}{|c|c|c|c|c|c|c|c|c|c|c|}
\hline \multirow{2}{*}{\multicolumn{2}{|c|}{$\begin{array}{c}\begin{array}{c}\text { Vergleich } \\
\text { der Dicke }\end{array} \\
\text { Material }\end{array}$}} & \multicolumn{3}{|c|}{$\mathrm{EZ}$} & \multicolumn{3}{|c|}{$\mathrm{ZZ}$} & \multicolumn{3}{|c|}{ Eiigkeit unklar } \\
\hline & & \multirow{2}{*}{$\begin{array}{l}\mathrm{L} \\
54 \\
32\end{array}$} & \multirow{2}{*}{$\begin{array}{l}\mathrm{R} \\
54 \\
27\end{array}$} & \multirow{2}{*}{$\begin{array}{c}\begin{array}{c}\text { Summe } \\
108\end{array} \\
59 \\
(54.6 \% \\
\pm 4.79)\end{array}$} & \multirow{2}{*}{$\begin{array}{r}\mathrm{L} \\
28 \\
20\end{array}$} & \multirow{2}{*}{$\begin{array}{l}\mathrm{R} \\
28 \\
\text { I } 9\end{array}$} & \multirow{2}{*}{$\begin{array}{c}\text { Summe } \\
56\end{array}$} & \multirow{2}{*}{$\begin{array}{r}\mathrm{L} \\
8 \\
\\
\end{array}$} & \multirow{2}{*}{$\begin{array}{c}\mathrm{R} \\
8 \\
6\end{array}$} & \multirow{2}{*}{$\begin{array}{c}\begin{array}{c}\text { Summe } \\
\text { I } 6\end{array} \\
10 \\
(62.5 \% \\
\pm 12.2)\end{array}$} \\
\hline \multirow{3}{*}{$\begin{array}{c}\text { Anastomose } \\
(+)\end{array}$} & Lat $>$ Med & & & & & & & & & \\
\hline & Lat $=$ Med & 7 & IO & $\begin{array}{c}17 \\
(15.8 \% \\
\pm 3.51)\end{array}$ & 4 & I & $\begin{array}{c}5 \\
(8.9 \% \\
\pm 3.80) \\
\end{array}$ & I & I & $\begin{array}{c}2 \\
(12.5 \% \\
\pm 8.26) \\
\end{array}$ \\
\hline & Lat $<$ Med & 7 & 2 & $\begin{array}{c}9 \\
(8.3 \% \\
\pm 2.55)\end{array}$ & 2 & 3 & $\begin{array}{c}5 \\
(8.9 \% \\
\pm 3.80)\end{array}$ & 3 & I & $\begin{array}{c}4 \\
(25.0 \% \\
\pm 10.8)\end{array}$ \\
\hline \multirow{2}{*}{$\begin{array}{c}\text { Anastomose } \\
(-)\end{array}$} & Lat $>$ Med & 2 & $\mathbf{x}$ & $\begin{array}{c}3 \\
(2.8 \% \\
\pm 1.59)\end{array}$ & o & $\mathbf{I}$ & $\begin{array}{c}\mathrm{I} \\
(\mathrm{x} .8 \% \\
\pm \mathrm{x} .35)\end{array}$ & 0 & $\circ$ & 一 \\
\hline & Lat $<$ Med & 6 & I 4 & $\begin{array}{c}20 \\
(18.5 \% \\
\pm 3.88) \\
\end{array}$ & 2 & 4 & $\begin{array}{c}6 \\
(10.7 \% \\
\pm 4.13)\end{array}$ & $\circ$ & o & - \\
\hline
\end{tabular}

$\mathrm{L}=$ links, $\mathrm{R}=$ rechts, $\mathrm{Lat}=\mathrm{N}$. cutaneus surae lateralis, $\mathrm{Med}=\mathrm{N}$. cutaneus surae medialis.

beiden Geschlechtern und Zwillingsarten auffindet. Wenn man nun, nach dem Geschlecht sowie der Eiigkeit gesehen, die Häufigkeit der symmetrischen und asymmetrischen Verteilung hinsichtlich der Dickenverhältnisse der bei- 
den Wadennerven in den einzelnen Feten berechnet, so unterrichtet hierüber die Tabelle 18 und 19. Diese Tabellen erhellen, dass bezüglich der Dickenverhältnisse die weiblichen Feten mehr asymmetrisch als die männlichen einerseits, die ZZ mehr asymmetrisch als die EZ anderseits geneigt sind. Verteilt man die 45 Zwillingspaare auf das Dickenverhältnis der beiden Wadennerven unter vier Beinen eines Paares, so ergibt sich die Tabelle 20. Daraus ersieht man, dass die Häufigkeit des gleichen Dickenverhältnis an vier Beinen bei EZ am grössten (15/27) ist, obwohl sie bei ZZ mit der Häufigkeit des gleichen Dickenverhältnis an drei Beine gerade gleich (s/14) ist.

Tabelle I8. Vergleich über die

Dickenverhältnisse der beiden

Wadennerven in den einzelnen

Individuen nach dem

Geschlecht.

\begin{tabular}{|c|c|c|}
\hline $\begin{array}{l}\text { Dicke der } \\
\text { W'aden- } \\
\text { nerven } \\
\text { Ge- } \\
\text { schlecht }\end{array}$ & $\begin{array}{l}\text { Symmetri- } \\
\text { sche Dicken- } \\
\text { verhältnisse }\end{array}$ & $\begin{array}{l}\text { Asymmetri- } \\
\text { sche Dicken- } \\
\text { verhältnisse }\end{array}$ \\
\hline (ss Feten) & $\begin{array}{l}43 \\
(78.2 \% \\
\quad \pm 5.56)\end{array}$ & $\begin{array}{l}12 \\
(21.8 \% \\
\pm 5.56)\end{array}$ \\
\hline (3s $\stackrel{\text { Feten) }}{\text { Fet }}$ & $\begin{array}{c}18 \\
(51.4 \% \\
\pm 8.44)\end{array}$ & $\begin{array}{c}17 \\
(48.6 \% \\
\pm 8.44)\end{array}$ \\
\hline
\end{tabular}

Tabelle 19. Vergleich über die Dickenverhältnisse der beiden Wadennerven in den einzelnen Individuen nach der Eiigkeit.

\begin{tabular}{|c|c|c|}
\hline $\begin{array}{c}\text { Dicke der } \\
\text { Waden- } \\
\text { nerven }\end{array}$ & $\begin{array}{c}\text { Symmetri- } \\
\text { sche Dicken- } \\
\text { verhältnisse }\end{array}$ & $\begin{array}{c}\text { Asymmetri- } \\
\text { sche Dicken- } \\
\text { verhältnisse }\end{array}$ \\
\hline $\begin{array}{c}\text { EZ } \\
(54 \text { Feten })\end{array}$ & $\begin{array}{c}38 \\
(70.4 \% \\
\pm 6.20)\end{array}$ & $\begin{array}{c}16 \\
(29.6 \% \\
\pm 6.20)\end{array}$ \\
\hline $\begin{array}{c}\text { ZZ } \\
(28 \text { Feten })\end{array}$ & $\begin{array}{c}(64.3 \% \\
\pm 9.05)\end{array}$ & $\begin{array}{c}10 \\
(35.7 \% \\
\pm 9.05)\end{array}$ \\
\hline
\end{tabular}

Tabelle 20. Vergleich über die Dicke der beiden Wadennerven beim einzelnen Zwillingspaar.

\begin{tabular}{|c|c|c|c|c|}
\hline \multicolumn{2}{|c|}{ Eiigkeit } & $\begin{array}{c}\text { EZ } \\
\text { Befund }\end{array}$ & $\begin{array}{c}\text { ZZ } \\
\text { (27 Paare) }\end{array}$ & $\begin{array}{c}\text { (14 Paare) } \\
\text { (4 Paare) }\end{array}$ \\
\hline Gleicher Typus an vier Beinen & Konkordanz & I & I & I \\
\hline Gleicher Typus an drei Beinen & Diskordanz & 5 & I & I \\
\hline $\begin{array}{c}\text { Gleicher Typus an zwei Beinen jeder } \\
\text { Körperseite }\end{array}$ & Konkordanz & 3 & I \\
\hline $\begin{array}{c}\text { Gleicher Typus an zwei Beinen jedes } \\
\text { Paarlings }\end{array}$ & $\begin{array}{c}\text { Diskordanz } \\
\text { Spiegelbildliche } \\
\text { Konkordanz }\end{array}$ & I & I & I \\
\hline $\begin{array}{c}\text { Spiegelbildlich gleicher Typus an } \\
\text { zweinen }\end{array}$ & $\begin{array}{c}\text { Diskordanz } \\
\text { Sonstige }\end{array}$ & 2 & I & I \\
\hline
\end{tabular}

Summieren wir zum Schluss unsere Ergebnisse hinsichtlich des Ähnlichkeitsgrades der Verteilung der Wadennerven bei den Zwillingspaaren, so haben wir die Tabelle 21, woraus wir den folgenden Schluss ziehen können. 
Tabelle 21. Ähnlichkeit der untersuchten Zwillingspaare.

\begin{tabular}{|c|c|c|c|c|c|c|}
\hline \multirow{2}{*}{ Alig- } & \multicolumn{2}{|c|}{$\begin{array}{l}\text { Über die Anastomose } \\
\text { der beiden Wadennerven }\end{array}$} & \multicolumn{2}{|c|}{$\begin{array}{c}\text { Über die Höhe der } \\
\text { Anatomose der beiden } \\
\text { Wadennerven }\end{array}$} & \multicolumn{2}{|c|}{$\begin{array}{l}\text { Uber die Dicke der } \\
\text { beiden Wadennerven }\end{array}$} \\
\hline & $\begin{array}{c}\text { EZ } \\
(27 \text { Paare) }\end{array}$ & $\begin{array}{c}Z Z \\
\text { (14 Paare) }\end{array}$ & $\begin{array}{c}\text { EZ } \\
\text { (17 Paare) }\end{array}$ & $\begin{array}{c}\mathrm{ZZ} \\
\text { (1。 Paare) }\end{array}$ & $\begin{array}{c}\text { EZ } \\
(27 \text { Paare) }\end{array}$ & $\begin{array}{c}\mathrm{ZZ} \\
\text { (14 Paare) }\end{array}$ \\
\hline Konkordanz & $\begin{array}{c}23 \\
(85.2 \% \\
\pm 8.85)\end{array}$ & $\begin{array}{c}\text { II } \\
(78.6 \% \\
\pm 10.95) \\
\end{array}$ & $\begin{array}{c}6 \\
(35.3 \% \\
\pm 11.59) \\
\end{array}$ & $\begin{array}{c}4 \\
(40.0 \% \\
\pm 15.47)\end{array}$ & $\begin{array}{c}18 \\
(66.7 \% \\
\pm 9.06) \\
\end{array}$ & $\begin{array}{c}6 \\
(42.9 \% \\
\pm 13.23)\end{array}$ \\
\hline $\begin{array}{l}\text { Spiegelbildliche } \\
\text { Konkordanz }\end{array}$ & $\begin{array}{c}1 \\
(3.7 \% \\
\pm 3.63)\end{array}$ & - & 一 & - & $\begin{array}{c}1 \\
(3.7 \% \\
\pm 3.63)\end{array}$ & $\begin{array}{c}1 \\
(7.1 \% \\
\pm 6.86)\end{array}$ \\
\hline Diskordanz & $\begin{array}{c}3 \\
(11.1 \% \\
\pm 6.05) \\
\end{array}$ & $\begin{array}{c}3 \\
(21.4 \% \\
\pm 10.95) \\
\end{array}$ & $\begin{array}{c}11 \\
(64.7 \% \\
\pm 11.59) \\
\end{array}$ & $\begin{array}{c}6 \\
(60.0 \% \\
\pm 15.47)\end{array}$ & $\begin{array}{c}8 \\
(29.6 \% \\
\pm 8.78)\end{array}$ & $\begin{array}{c}7 \\
(50.0 \% \\
\pm 13.35)\end{array}$ \\
\hline
\end{tabular}

I. Über das Auftreten der Anastomose zwischen den beiden Wadennerven ist die Häufigkeit der Konkordanz $7.7 \mathrm{mal}$ (bei EZ) oder $3.7 \mathrm{mal}$ (bei ZZ) grösser als die der Diskordanz.

2. Über die Höhe der Anastomose der beiden Wadennerven ist, ganz umgekehrt, die Häufigkeit der Diskordanz r.8 mal (bei EZ) oder I.5 mal (bei ZZ) grösser als die der Konkordanz.

3. Über die Dicke der beiden Wadennerven ist die Häufigkeit der Konkordanz 2.3 mal grösser als die der Diskordanz bei EZ, obwohl die der Konkordanz und Diskordanz bei ZZ annährend gleich ist.

Weiterhin lassen sich aus dem oben Gesagten die Unterschiede der Befunde zwischen den Zwillingen (Feten) und den Einlingen (Erwachsene) folgendermassen zusammenfassen.

I. Die Anastomose $z$ wischen den beiden Wadennerven trifft man häufiger bei Zwillingsfeten als bei Erwachsenen (Ssokolow, Kosinski und Catania).

2. Ssokolow hat die Anastomose in der Kniekehle in einem höheren Prozentsatz ( I 1.0\%) beobachtet, im Gegensatz zu meinen Fällen (1.3\%).

3. Bei den Zwillingsfeten ist die Häufigkeit derjenigen Nervenverteilung am grössten $(60.0 \%)$ : d.h. , die beiden Wadennerven anastomosieren miteinander, wobei der N. cut. surae lat. stärker als der N. cut. surae med. ist ". Bei den Einlingen (Erwachsenen, Ssokolow) ist die Häufigkeit derjenigen Nervenverteilung am grössten (43.8\%): d.h. "die N. cut. surae med. ist stärker als der N. cut. surae lat., ohne eine Anastomose dazwischen “.

Es ist wohl berechtigt, zu glauben, dass der Grund dieser Verschiedenheiten z.T. in dem Alterunterschied beruht. Wenn man das Ergebnis der späteren gleichen Arbeit bei Säuglingen oder Kindern mit dem der vorliegenden Arbeit vergleichend betrachten würde, so dürfte diese Frage einer Lösung nähergeführt sein. Unsere Untersuchung an einem grösseren Material von 
Zwillingsfeten sind noch im Gange, um mit dem Befund bei den Einlingsfeten als Kontrolle vergleichend, die Lösung der Frage, „, in wieweit die erbliche Beziehung dabei eine Rolle spielt?", näherzubringen. Wir sind heute also noch nicht in der Lage, einen bindenden Schluss über diese Frage daraus zu ziehen.

\section{Schluss.}

Fasst man die Hauptresultate der vorliegenden Untersuchung kurz zusammen, so ergibt sich folgendes :

I. Das Untersuchungsmaterial sind 45 paarige Zwillinge (EZ 27 Paare, ZZ I 4 Paare und 4 Paare von unklarer Eiigkeit), d.h. 90 Feten (ㅎ 55, 우 35).

2. Die Häufigkeit der Anastomose zwischen den beiden Wadennerven.

I) Die Anastomose kommt in $83.3 \% \pm 2.78$ vor, wobei sie links $(88.9 \% \pm 3.3 \mathrm{I})$ etwas häufiger als rechts $(77.8 \% \pm 4.36)$ zu sein scheint.

2) Die Häufigkeit scheint von dem Geschlecht oder Eiigkeit nicht wesentlich beeinflusst $\mathrm{zu}$ werden, indem sie bei $585.5 \% \pm 3.36$ und bei 우 $80.0 \% \pm 4.77$ einerseits, bei EZ $78.7 \% \pm 3.94$ und bei ZZ $87.5 \% \pm 4.4 \mathrm{I}$ andererseits ausmacht.

3) Hinsichtlich der Anastomose der beiden Wadennerven, tritt die asymmetrische Verteilung an einzelnen Feten bei 우 $(34.3 \% \pm 8.00)$ bedeutend häufiger als bei $\hat{o}(7.3 \% \pm 3.5 \mathrm{I})$ hervor, die aber zwischen $\mathrm{EZ}(24 . \mathrm{I} \% \pm 5.8 \mathrm{I})$ und $\mathrm{ZZ}(10.7 \% \pm 5.84$ ) keinen statistischen Unterschied aufweist.

4) Vergleicht man die vier Beine eines Zwillingspaares bezüglich der Anastomose, so findet man die konkordanten Paare in $85.2 \% \pm 8.8 \mathrm{~s}$ bei EZ und in $-8.6 \% \pm 10.95$ bei $\mathrm{ZZ}$.

3. Die Höhe der Anastomose zwischen den beiden Wadennerven.

I) Was die Höhe der Anastomose anbelangt, so kommt das mittlere Drittel des Lnterschenkels $(57.4 \% \pm 4.03)$ am häufigsten vor, danach folgt das untere Drittel $(28.0 \% \pm 3.66)$; die sonstige Höhe auf der Wade ist am wenigsten anzugeben. Diese Reihenfolge gilt für die Ergebnisse aller nach Körperseite, Geschlecht oder Eiigkeit eingeteilten Beobachtungen, wobei allein bemerkenswert ist, dass die Vereinigungsstelle der Nerven bei 우 mehr distal als bei ô sich zu verschieben geneigt ist.

2) Hinsichtlich der Höhe der Anastomose, tritt die asymmetrische Verteilung an den einzelnen Feten in $37.8 \% \pm 7.22$ bei $\hat{o}$ und $27.3 \% \pm 9.48$ bei o einerseits, und in $36.1 \% \pm 7.68$ bei EZ und $30.4 \% \pm 9.59$ bei $Z Z$ hervor.

3) Vergleicht man die vier Beine eines Zwillingspaares bezüglich der Höhe der Anastomose, so findet man die konkordanten Paare in 35.3\% t II. 59 bei EZ und in $40.0 \% \pm 15.47$ bei $\mathrm{ZZ}$.

4. Die Dicke der beiden Wadenerven. 
I) Es ist besonders hervorzuheben, einen solchen Fall mit der Anastomose am häufigsten zu treffen, wo der N. cutaneus surae lateralis dicker als der N. cutaneus surae medialis ist $(60.0 \% \pm 3.65)$. Beim Fall ohne Anastomose ist dagegen der N. cut. surae med. fast immer (I $4.5 \% \pm 2.63 \%$ ) dicker als der N. cut. surae lat. Das obige Verhältnis gilt für die Ergebnisse aller nach Körperseite, Geschlecht oder Eiigkeit eingeteilten Beobachtungen.

2) Hinsichtlich der Dicke der Anastomose, kommt die asymmetrische Verteilung an den einzelnen Feten bei 우 (48.6\% 18.44$)$ häufiger als bei ô $(21.8 \% \pm 5.56)$ vor, die aber zwischen EZ (29.6\%土6.20) und ZZ $(35.7 \% \pm 9.05)$ keinen statistischen Unterschied aufweist.

3) Vergleicht man die vier Beine eines Zwillingspaares bezüglich der Dicke der Wadennerven, so findet man die konkordanten Paare in $66.7 \% \pm$ 9.06 bei $\mathrm{EZ}$ und in $42.9 \% \pm 13.23$ bei $\mathrm{ZZ}$.

5. In dieser Untersuchung habe ich zufällig eine seltene, noch von niemandem beschriebene Varietät der Nervenverteilung der Wade gefunden, wo der fortsetzende $N$. cutaneus femoris posterior und der $N$. cutaneus surae medialis, miteinander anastomosierend, an der Innervation der Wade sich beteiligen (es fehlt der $N$. cutaneus surae lat.).

Am Schlusse spreche ich Herrn Professor T. Taniguchi für seine stete liebenswürdige Unterstützung bei dieser Arbeit meinen herzlichsten Dank aus.

\section{Literatur.}

I. Andreassi, G., Osservazioni intorno all' origine, comportamento e distribuzione dei nervi cutaneo mediale della sura, ramo anastomotico peoniero e cutaneo laterale della sura nell' uomo (Ist. Anat. U. Nor. R. Univ. Roma). Ric. morf. II. I93 I (Referat).

2. Bardeleben, C. R., Development and variation of the nerves and the musculature of the inferior extremity and of the neighbouring regions of the trunk in man. The Americ. Journ. of Anat. Vol. VI. 1906-1907.

3. Catania, V., Il comportamento dei nervi cutanei dorsali del piede (Ricerche statistiche nei Siciliani) Arch. Ital. Anat. Embriol. 21 . 1924 (Referat).

4. Corning, H. K., Lehrbuch der topographischen Anatomie. 1923.

5. Gegenbaur, C., Lehrbuch der Anatomie des Menschen Bd. II. I899.

6. Kosinski, A., The course, mutual relations and distribution of the cutaneaus nerves of the metazonal region of leg and foot. Journ. of Anat. Vol. LX. 1926.

7. Lubosch, W., Topographische Anatomie. 4. Auflage. 1935.

8. Rauber-Kopsch, Lehrbuch der Anatomic des Menschen. 1930.

9. P' an Ming-Tzu, The cutaneous nerves of the chinese foot. Chin. Med. Journ. $48 . \quad$ 1934.

10. Spaltholz, W., Handatlas der Anatomie des Menschen. Bd. 3. 1935.

r1. Ssokolow, P.A., Zur Anatomie des N. suralis beim Menschen und Affen. Zeitschr. $\mathrm{f}$. Anat. u. Entw.-gesch. Bd. 100. 1933.

I2. Tandler, J., Lehrbuch der systematischen Anatomie. Bd. 4. Nervensystem u. Sinnesorgane. I929.

13. Thomson, A., A comparatively small number of observations has been made on the distribution of the cutaneous nerves to the dorsum of the foot. Journ. of Anat. and Physiol. Vol. XXVI. I 892.

I4. Toldt-Hochstetter, Anatomischer Atlas. Bd. III. I93I.

Is. Ushio, S., Úber die Verteilung der Hautnerven an den unteren Extremitäten bei den Japanern. Japan. Zeitschr. f. Anat. Bd. 7. I 934 (Japanisch). 
Fig. I. Fall I (EZ).

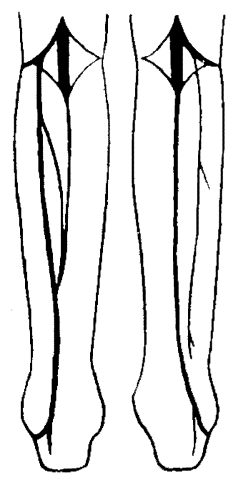

Nr. 168 (I).

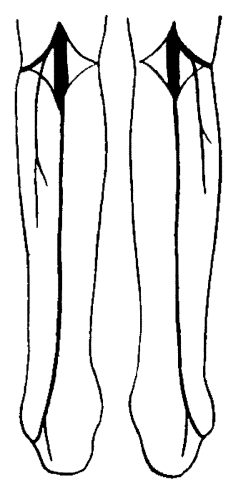

Nir. 168 (II)

Fig. 2. Fall 2 (EZ).

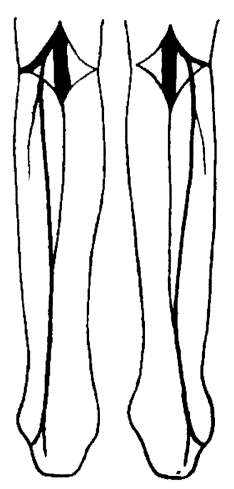

Nr. 228 (I).

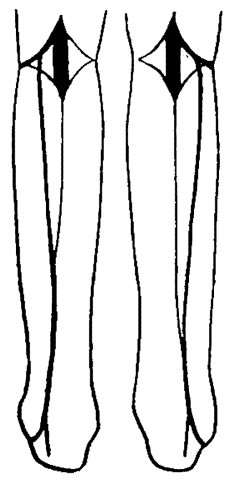

Nr. 228 (II).

Fig. 3. Fall 3 (EZ).

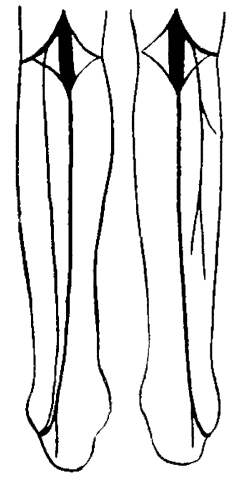

Nit. 322 .

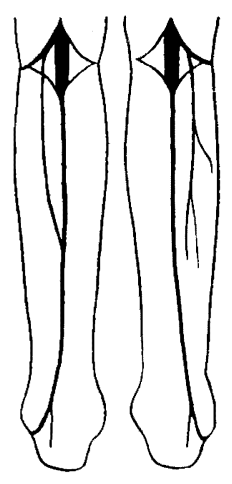

Nr. 323 .

Fig. 4. Fall 4 (EZ).

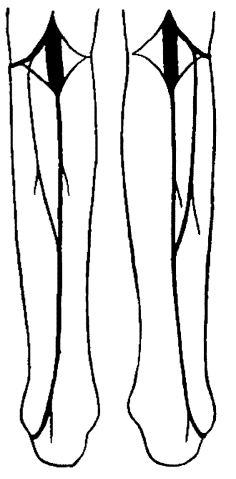

Nr. 399.

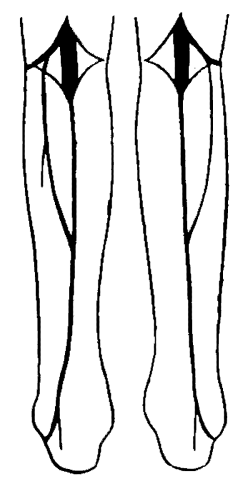

Nr. 400.

Fig. 5. Fall s (EZ).

Fig. 6. Fall 6 (EZ).

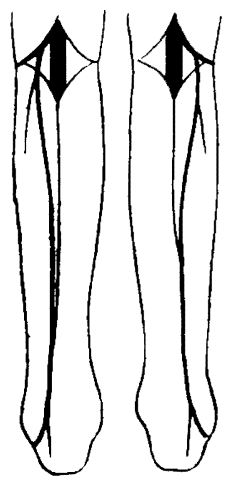

Nir. 566.

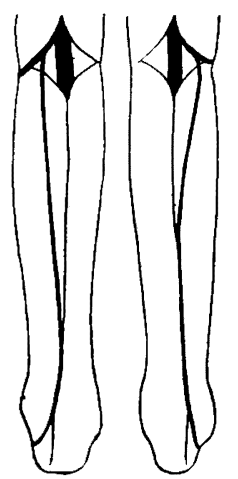

Nr. 567.

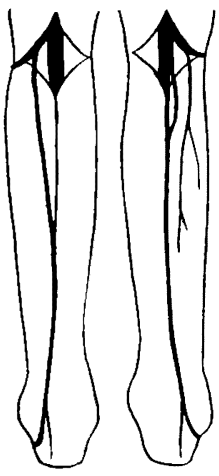

Nr. 734

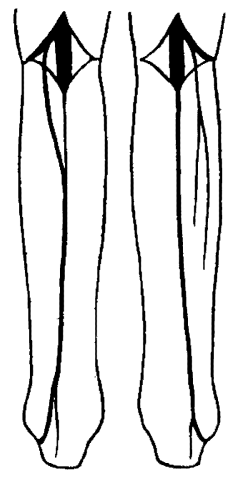

Nr. 735 . 
Fig. 7. Fall 7 (EZ).

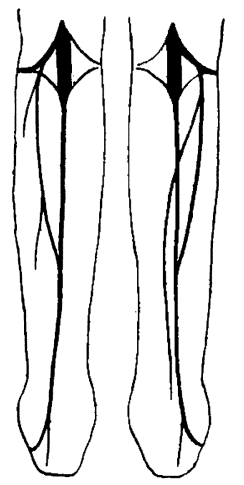

Nr. 768.

Fig. 9. Fall 9 (EZ).

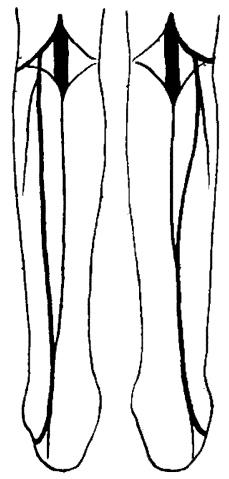

Nr. IOI 2.

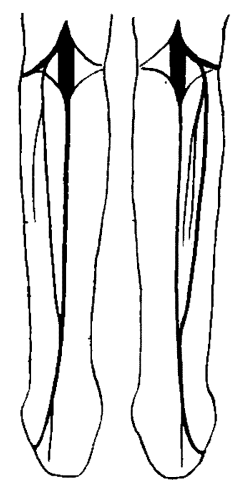

Nr. 770.

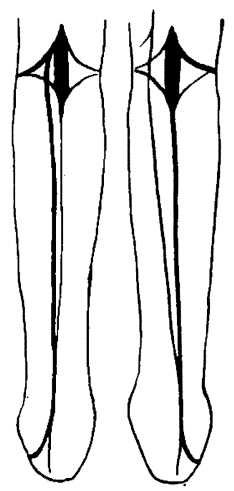

Nr. 825 .
Fig. 8. Fall 8 (EZ).

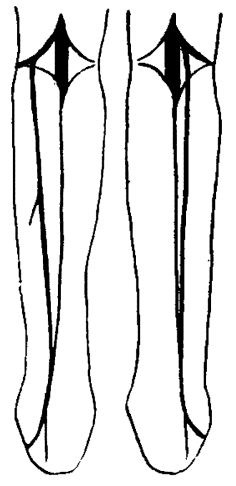

Nr. 826.

Fig. 1o. Fall ro (EZ).

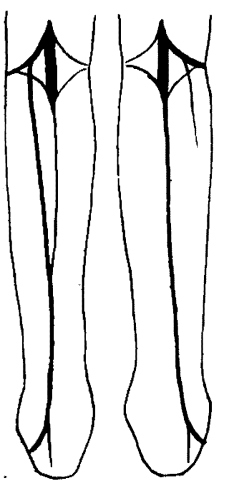

Nr. ${ }_{0} 063$.

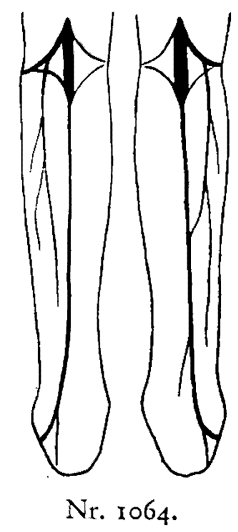

Fig. I 2. Fall I 2 (EZ).

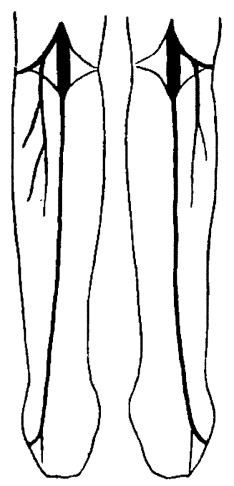

Nr. 1254.

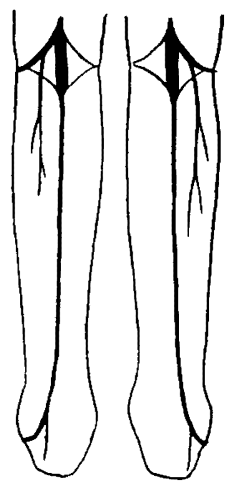

Nr. 1255 . 
Uber die sensiblen Wadennerven bei den japanischen Zwillingen.

Fig. 13. Fall $\mathrm{I}_{3}$ (EZ).

Fig. 14. Fall i4 (EZ).

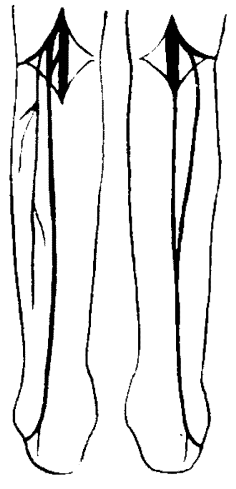

Nr. 126 r.

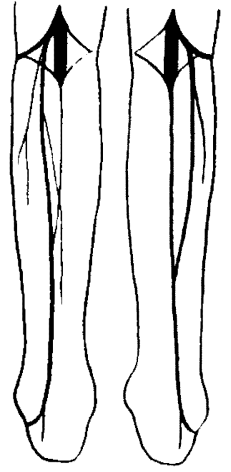

Nr. 1262.

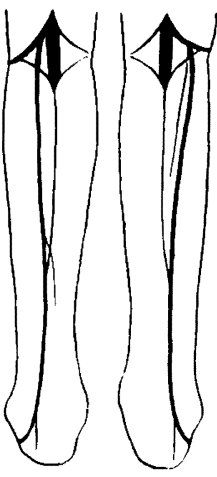

Nr. 1300.

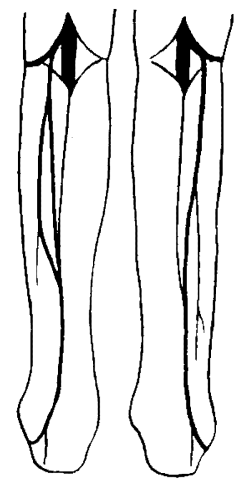

Nr. I30x.

Fig. Is. Fall is (EZ).
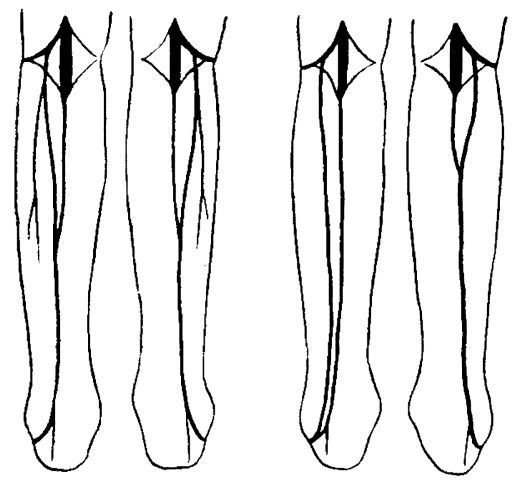

Nr. 1425 .

Fig. 17. Fall 17 (EZ).

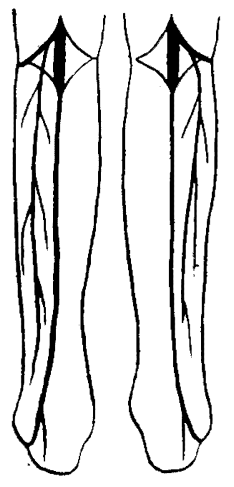

Nr. 1445 .

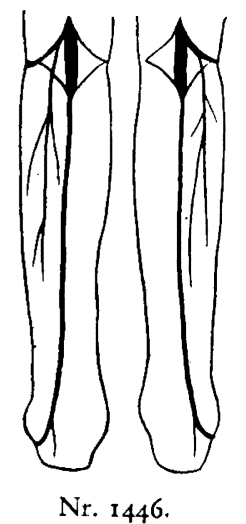

Fig. 16. Fall 16 (EZ).

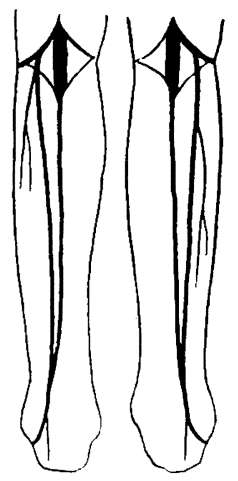

N.. 143 I.

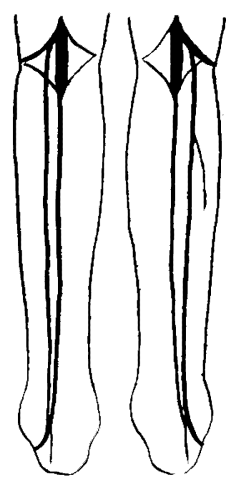

Nr. 1432.

Fig. 18. Fall 18 (EZ).
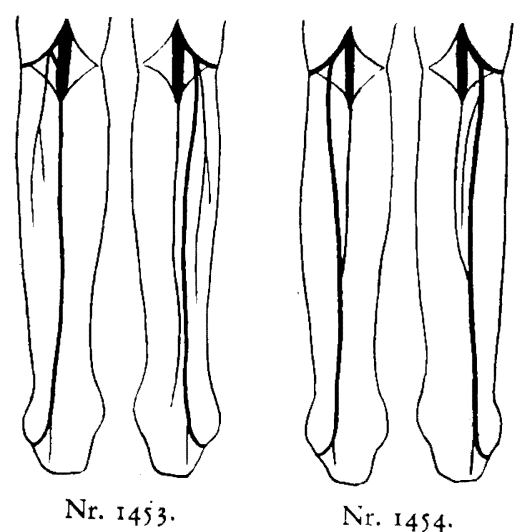
Fig. 19. Fall I9 (EZ).
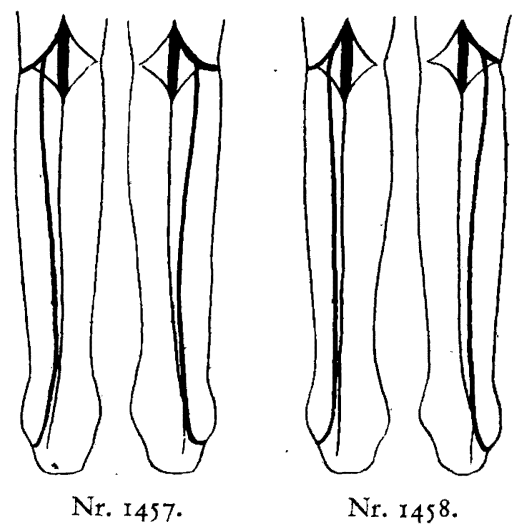

Fig. 21. Fall 2I (EZ).

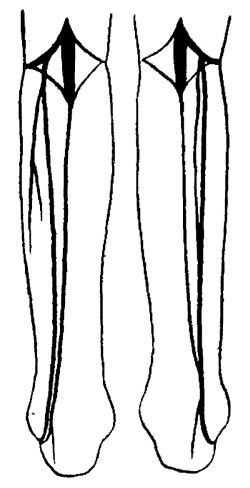

Nr. $146 r$.

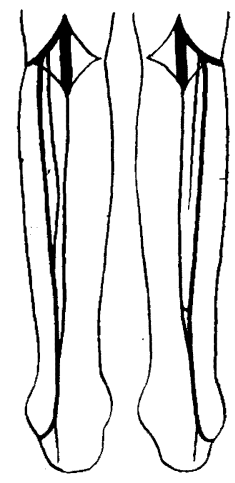

Nr. 1462 .

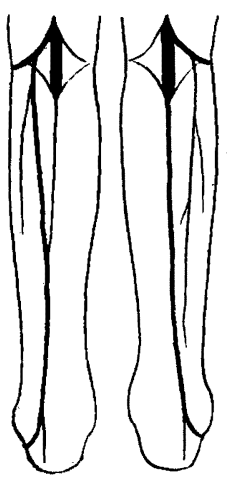

Nr. 1459.

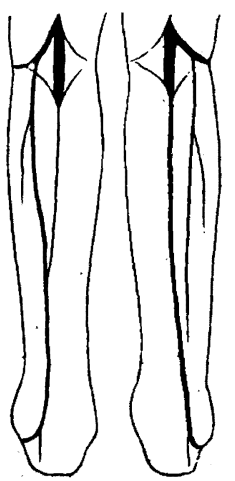

Nr. 1460.

Fig. 22. Fall 22 (EZ).
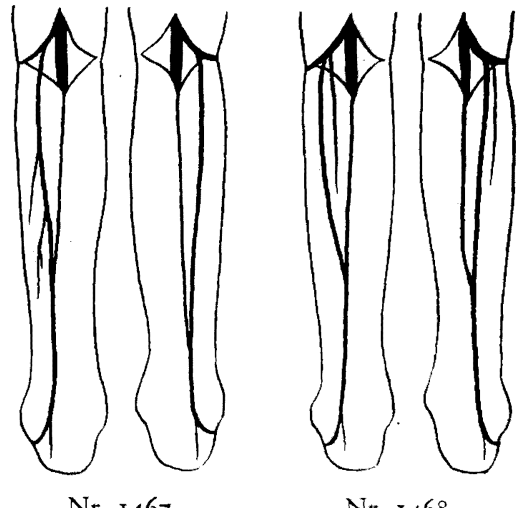

Nr. 1468.

Fig. 23. Fall 23 (EZ).

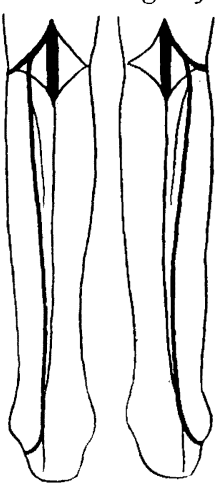

Nr. 1469 .

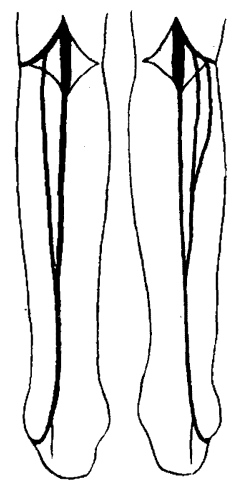

Nr. 1470 .

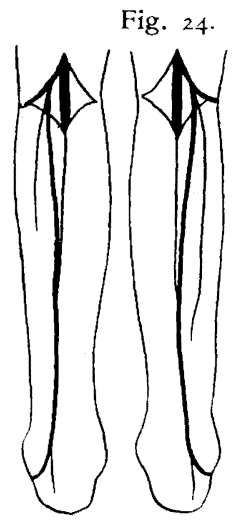

Nr. 1475 .

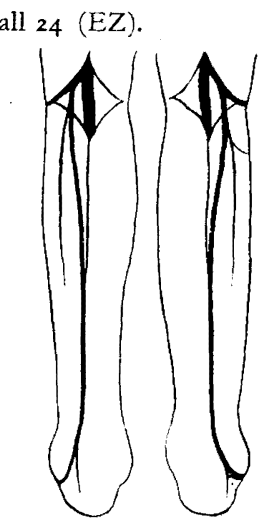

Nr. 1476 . 
Fig. 25. Fall 25 (EZ)

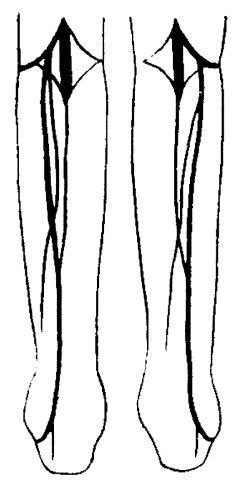

Nir. 1477.

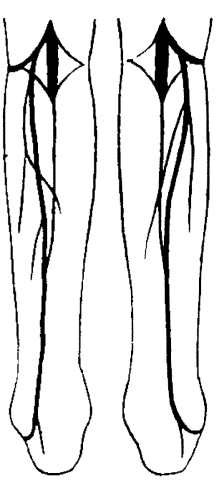

Nr. 1478 .

Fig. 27. Fall 27 (EZ).

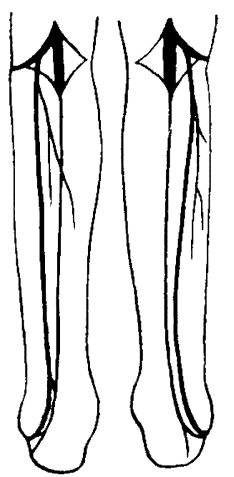

Nr. I520,

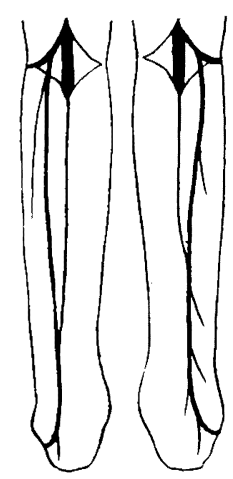

Nr. IS2I.

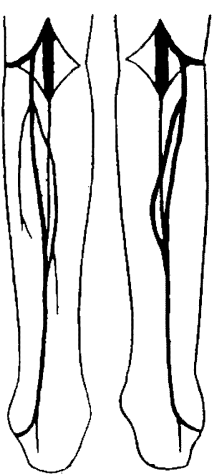

Nr. Is 14.

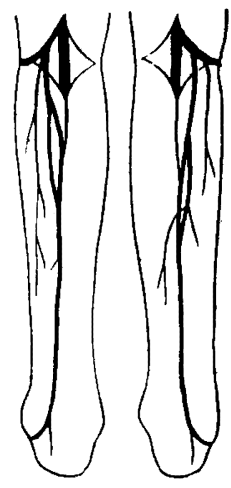

Nr. I5I5.

Fig. 28. Fall $28(\mathrm{ZZ})$.

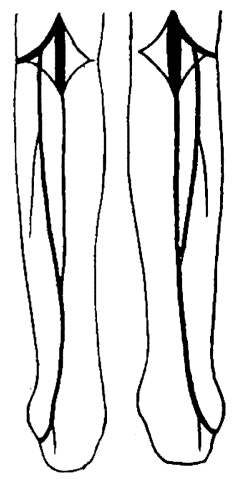

N.: 762.

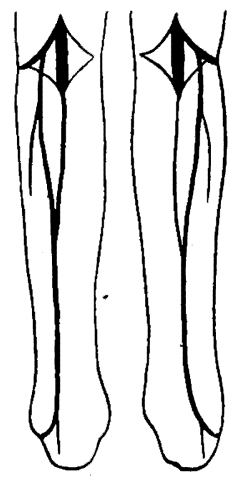

Nr. 763 .

Fig. 30. Fall 30 (ZZ).

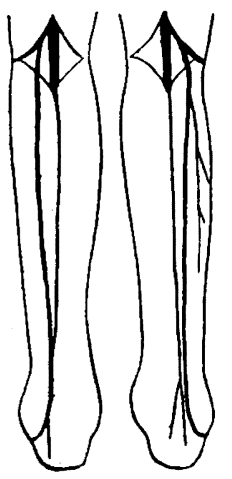

Nr. 954.

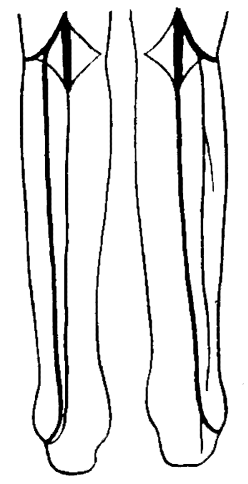

Nr. 955.

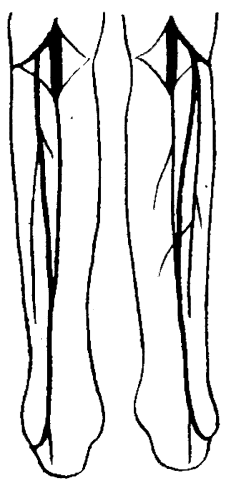

Nr. 765 .

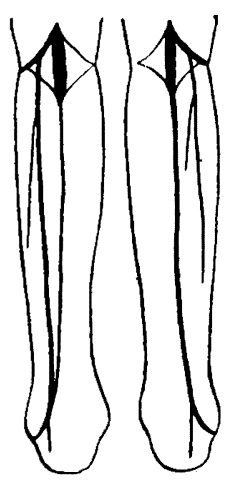

Nir. 769 . 
Fig. 3I. Fall 3I (ZZ).

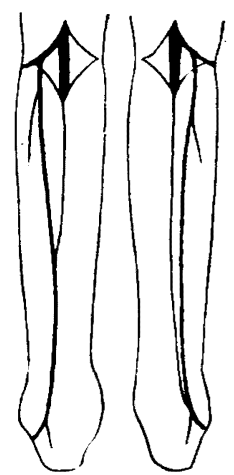

Nr. 961.

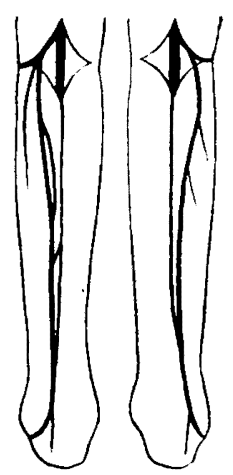

Nr. 962.

Fig. 33. Fall 33 (ZZ).
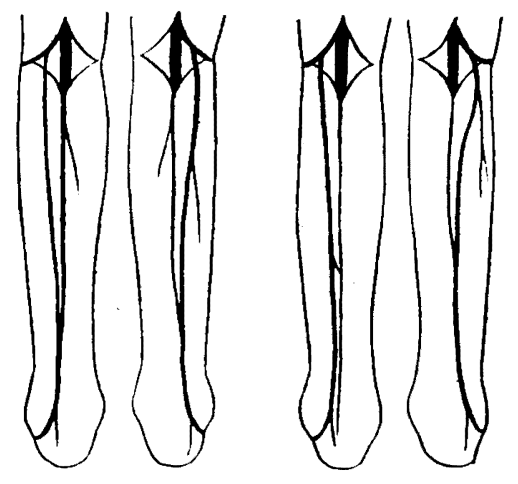

Nr. Ir6s.

Nr. 1166.

Fig. 35. Fall 35 (ZZ).
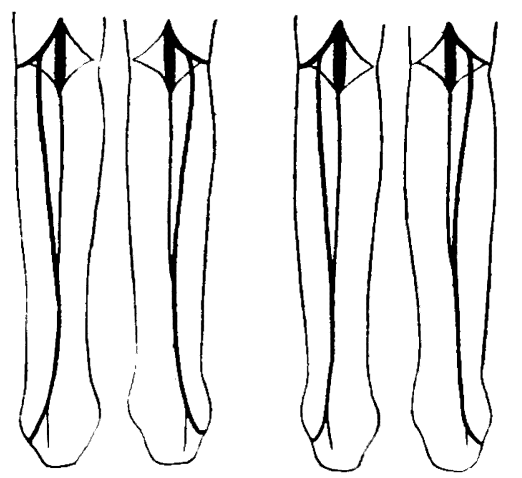

Nr. 1349.

Nr. 1350.

Fig. 32. Fall $32(\mathrm{ZZ})$.
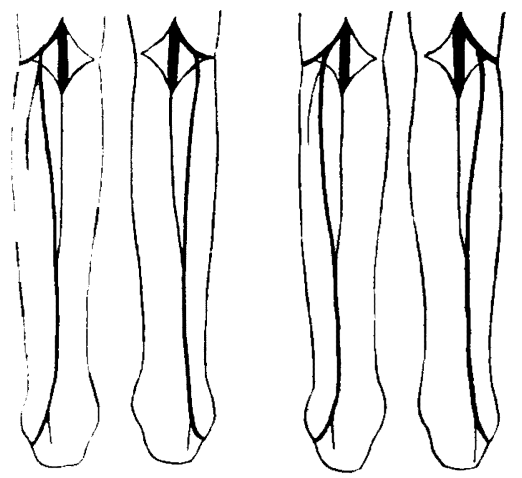

Nr. 1080.

Nr. 1081 .

Fig. 34. Fall 34 (ZZ).

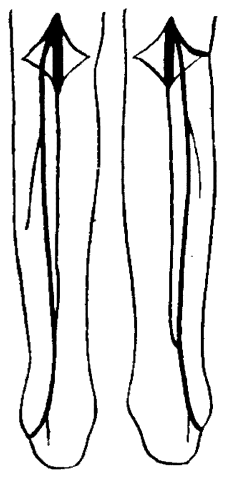

Nr. 1323.

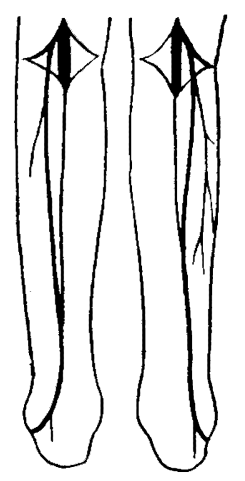

Nr. I324.

Fig. 36. Fall $36(\mathrm{ZZ})$.

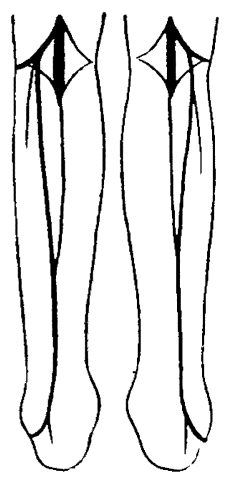

Nr. 1426 .

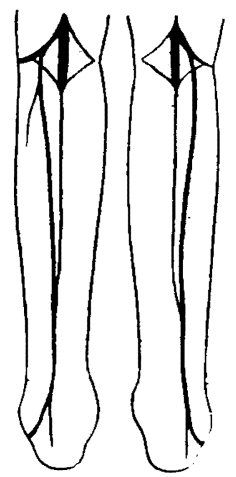

Nr. 1427. 
Uber die sensiblen Wadennerven bei den japanischen Zwillingen.

Fig. 37. Fall $37(\mathrm{ZZ})$.
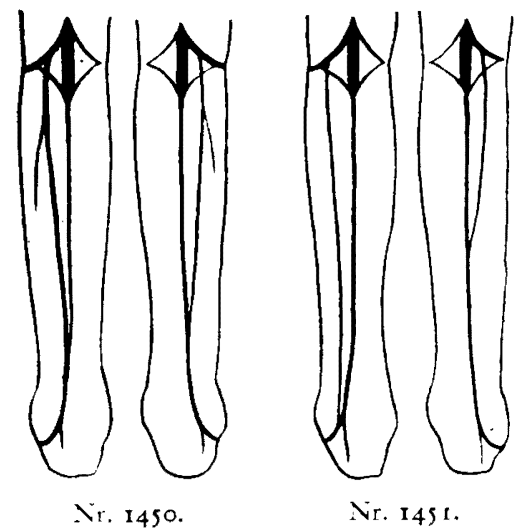

Fig. 39. Fall 39 (ZZ).

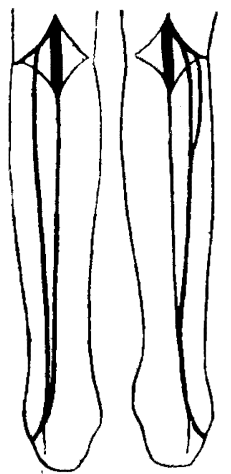

ㄱ. 1465 .

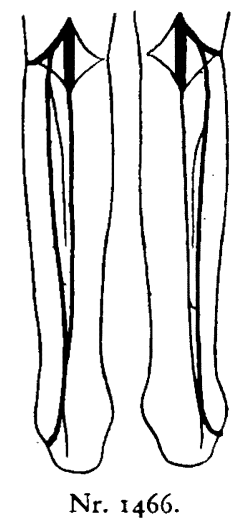

Fig. 4I. Fall 4I (Eiigkeit unklar).
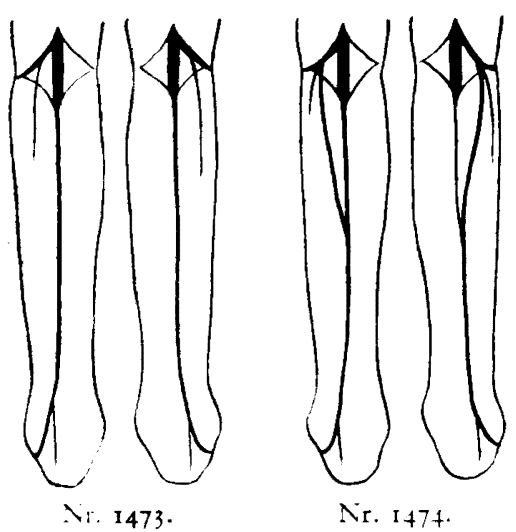

Fig. 38. Fall $3^{8}$ (ZZ).

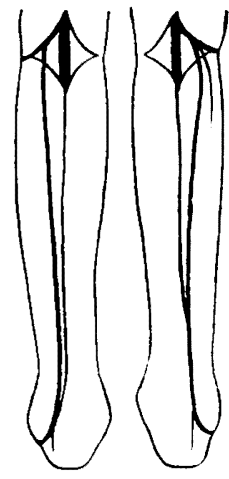

Nr. 1463 .

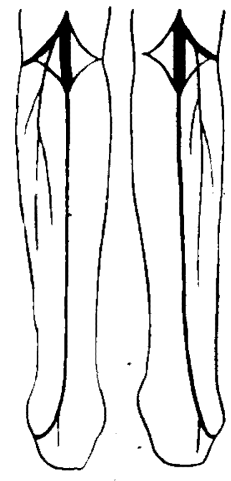

Nr. 1464 .

Fig. 40. Fall $40(\mathrm{ZZ})$.

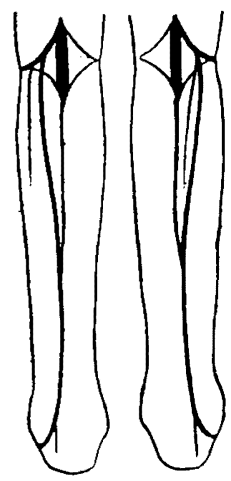

Nir. 1471.

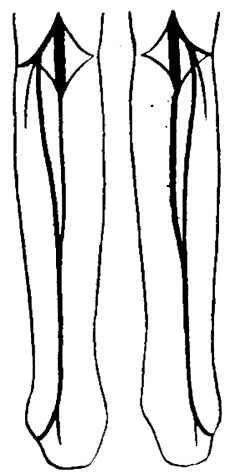

Nr. 472 .

Fig. 42. Fall 42 (Eiigkeit unklar).

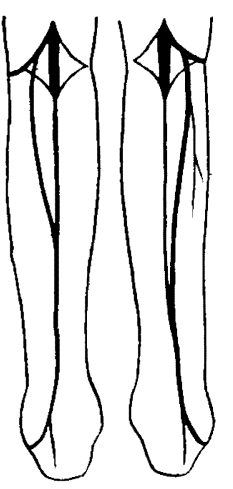

Nr. 540.

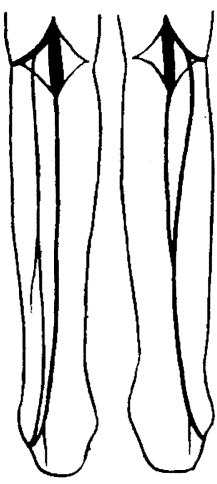

Nr. 54I. 
Fig. 43. Fall 43 (Eiigkeit unklar).

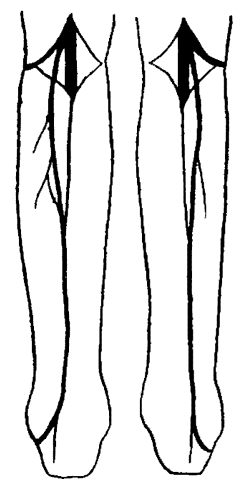

Nr. 57 I.

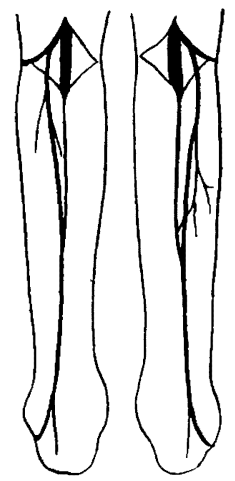

Nr. 572 .
Fig. 44. Fall 44 (Eiigkeit unklar).

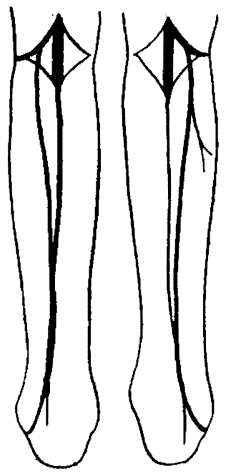

Nr. 1003.

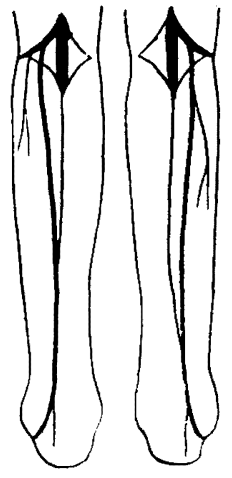

Nr. 1004 .

Fig. 45. Fall 45 (Eiigkeit unklar).

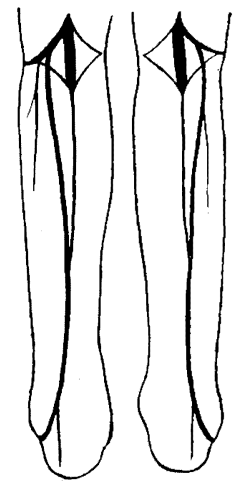

Nr. IIOS (I).

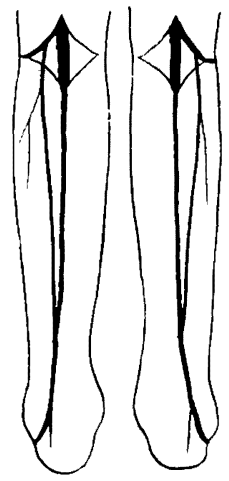

Nr. IIOS (II). 